\title{
TRANSFER FUNCTION CONCEPT FOR ULTRASONIC CHARACTERIZATION OF MATERIAL MICROSTRUCTURES
}

\author{
Alex Vary and Harold E. Kautz \\ National Aeronautics and Space Administration \\ Lewts Research Center \\ Cleveland, Oh10 44135
}

Ultrasonic attenuation measurements provide nondestructive means for characterizing material properties. In polycrystalline solids the frequency dependence of attenuation characterizes microstructure and hence the mechanical properties governed by microstructure. Microstructure, morphology, specific mechanical properties, and ultrasonic characteristics of materials are often uniquely interrelated (Green, 1973a, 1981, 1985; Vary, 1984).

Although expressions that relate ultrasonic attenuation to specific microstructural factors exist, they are fragmented and unsatisfactory because they consist of diverse piecemeal descriptions and ad hoc combinations of attenuation mechanisms over various frequency ranges of interest (Merkulov, 1956; Papadakis, 1960, 1965b; Serabian and Williams, 1978). A unified approach is needed to overcome these shortcomings and to help resolve ques... tions that arise in attempts to identify and evaluate key microstructural factors that govern mechanical properties.

The approach given herein depends on treating material microstructures as elastomechanical "filters" that have analytically definable transfer functions. These transfer functions can be defined in terms of the frequency dependence of the ultrasonic attenuation coefficient (Vary, 1980b, 1984). The transfer function concept provides a basis for synthesizing expressions that characterize polycrystalline materials relative to microstructural factors such as mean grain size, grain-size distribution functions, and grain boundary energy transmission. Although our approach is nonrigorous, it leads to a rational basis for combining the previously mentioned diverse and fragmented equations for ultrasonic attenuation coefficients.

\section{BACKGROUND AND THEORY}

\section{General}

Ultrasonic materials characterization involves both velocity and attenuation measurements. Velocity measurements are useful for measuring elastic constants, residual stresses, density, etc. This paper concentrates on attenuation measurements because they are pivotal to understanding interrelations between microstructure and mechanical properties. The characterization of mechanical properties depends on precise measurements of attenuation.

U1trasonic frequency-spectrum analysis provides a means for characterizing microstructural factors that govern mechanical properties (Papadakis, 1976; Fitting and Adler, 1981; Vary, 1984). The frequency range emphasized 
herein is that normally used for interrogating polycrystalline engineering materials (i.e., about 10 to $100 \mathrm{MHz}$ ). By using ultrasonic frequency-spectrum analysis and signal deconvolution algorithms (Bracewell, 1978; Bendat and Pierso1, 1980; Vary, 1980b; Williams, et a1., 1985a), it is possible to define transfer functions that characterize microstructures relative to their effect on wave propagation and also on mechanical properties. It will be seen that these transfer functions are definable in terms of the frequency dependence of the attenuation coefficient.

\section{Pulse-Echo Method}

F'or the purposes of this paper we assume a material sample with flat, parallel opposing surfaces. The sample is examined with a broadband, pulseecho system using digital signal acquisition and processing (fig. 1). A buf fered, piezoelectric transducer collects a set of echoes returned by the back surface of the sample. Only the first two echoes, $B_{1}$ and $B_{2}$, are selected for analysis. Signal acquisition, processing, and analysis are done as described by Papadakis (1976), Vary (1979b, 1980a,b), Generazio (1984), and Kline (1984). The echoes are transformed to the frequency domain by digital Fourier transform algorithms. Ultrasonic attenuation as a function of frequency and therefore information on material microstructure is obtained by deconvolution of echoes $B_{1}$ and $B_{2}$.

\section{Material Transfer Function}

In the following discussion the quantities $B_{1}, B_{2}, I_{1}, I_{2}, T$, and $R$ (fig. 2) are Fourier transforms of corresponding time-domain quantities. The quantities $B_{1}, B_{2}, I_{1}$, and $I_{2}$ are spectra of waveforms, and $T$ is the material transfer function, to be defined in the frequency domain. (Phase spectra and such associated topics as waveform synthesis and phase interference effects are not considered herein.) The reflection coefficient $R$ is also a function of frequency, albeit a weak function in most cases (Papadakis, 1968; Generazio, 1984). The transfer function will be defined in terms of ${ }^{\mathrm{B}_{1}}, \mathrm{~B}_{2}$, and $\mathrm{R}$.

Internal echo $I_{1}$ is the source of the "reduced" signal $B_{1}$ (i.e., reduced by the factor $(1+R)$, where $R$ is the reflection coefficient at the specimen transducer interface). The reflection coefficient is unity (i.e., 1) at the free surface. Algebraic signs carried by reflection coefficients depend on whether they are defined as power or amplitude quantities and on the input-output direction of signals across interfaces. The "power" (energy) definition as given in appendix $A$ applies to $R$ herein. A part of the energy of $I_{1}$ is reflected and appears as the second internal echo $I_{2}$ giving the reduced echo $\mathrm{B}_{2}$; thus

$$
\begin{aligned}
& \mathrm{B}_{1}=(1-\mathrm{R}) \mathrm{I}_{1} \\
& \mathrm{~B}_{2}=\operatorname{TR}(1-\mathrm{R}) \mathrm{I}_{1}
\end{aligned}
$$

where $B_{1}, B_{2}$, and $I_{1}$ are the "power" (i.e., magnitude-squared spectra of corresponding amplitude squared time-domain signals). Combining equations (1) and (2) yields 


$$
T=\frac{\mathrm{B}_{2}}{\mathrm{RB}_{1}}
$$

There are transfer functions associated with the instrumentation, signal transduction, and other aspects of the signal acquisition system. These can be ignored because in the pulse-echo configuration they vanish, just as the term $(1+R) I_{1}$ vanishes in equation ( 3$)$.

\section{Attenuation Coefficient}

Papadakis (1968; 1976) has demonstrated that the attenuation coefficient can be found by frequency spectrum analysis and that it may be written as

$$
\alpha=\left(\frac{1}{2 x}\right) \ln \left(\frac{\mathrm{RB}_{1}}{\mathrm{~B}_{2}}\right)
$$

where $x$ is the sample thickness. The attenuation coefficient $\alpha$ is a function of frequency and hence as with the quantities $T, B_{1}$, and $B_{2}$, it is appropriate to treat it as a Fourier transform of a time-domain quantity. Then because the transfer function $T$ and the attenuation coefficient $\alpha$ are both defined in terms of $B_{1}, B_{2}$, and $R$, equations ( 3 ) and (4) can be combined to get

$$
T=\exp (-2 x \alpha)
$$

Thus the transfer function is defined in terms of an attenuation coefficient. Conversely, if the material transfer function $T$ can be prescribed apriori, appropriate expressions can be found for the attenuation coefficient $\alpha$. It will be seen that a general, unified expression can be derived for $\alpha$ for the various energy loss mechanisms in polycrystalline solids by using the preceding equations and the transfer function concept.

\section{Attenuation Mechanisms}

The energy loss mechanisms that govern ultrasonic attenuation in the frequency ranges (bandwidths) of interest for polycrystalline engineering solids are known (Mason and McSkimmin, 1947; Lifsitz and Parkomovskii, 1950; Granato and Luicke, 1956; Merkulov, 1957; True11, et al., 1969; Bahtia, 1976). The two principal causes of attenuation are scattering and absorption. Diffusion, Rayleigh, and stochastic (or phase) scattering losses are extrinsic; absorption losses from dislocation damping, anelastic hysteresis, relaxation, and thermoelastic effects are intrinsic to individual grains (i.e., crystallites).

We are not concerned with geometrical losses, such as those due to diffraction effects, which are not inherent to material microstructures. These effects can be either controlled or eliminated from attenuation measurements by wel1-known experimental and data reduction procedures (Papadakis, 1975) in order to get the true attenuation coefficient as a function of frequency. 
Scattering usually accounts for the greatest portion of losses in polycrystalline aggregates (Papadakis, 1964a,b, 1965a,b, 1970; Serabian and Williams, 1976; Goebbels, 1980). For polycrystalline aggregates expressions that have been developed for the scatter attenuation coefficient $\alpha$ as a function of frequency $f$ are referred to three regimes that are defined by the ratio of mean grain size a to dominant wavelength $\lambda$.

For the Rayleigh scattering regime, where $\lambda \gg \pi a$,

$$
\alpha_{r}=c r^{a^{3} f^{4}}
$$

For the stochastic (phase) scattering regime, where $\lambda \cong \pi a$,

$$
\alpha_{p}=c_{p} a f^{2}
$$

For the diffusion scattering regime, where $\lambda \ll \pi a$,

$$
\alpha_{\mathrm{d}}=\mathrm{c}_{\mathrm{d}^{\mathrm{a}}}
$$

In equation (6) $a^{3}$ represents grain volume, where the grains are to be considered as having a variety of nonequiaxed, nonspherical shapes (Papadakis, $1965 \mathrm{~b}$ ). The constants $c$ contain other quantities (i.e., geometric factors, longitudinal and transverse velocities, density, and elastic anistropy factors)(Mason and McSkimmin, 1947, 1948; Lifsitz and Parkomovskii, 1950;

Merkulov, 1957; Truell, et al., 1969). These factors will be considered later in this paper.

\section{Intrinsic Mechanisms}

Absorption losses due to dislocation damping, hysteresis, thermoelastic effects, etc., are intrinsic to grains (crystallites) and involve direct conversion of acoustic energy to heat. These attenuation mechanisms are essen-tially independent of grain size, shape, volume, etc.

For hysteresis losses (Kolsky, 1953; Nowick and Berry, 1972)

$$
\alpha_{h}=c_{h} f
$$

For thermoelastic losses (Lucke, 1956)

$$
\alpha_{t}=c_{t} f^{2}
$$

Hysteresis losses (eq. (9)) arise when acoustic waves cause stress-strain damping. Hysteresis losses with a first-power frequency dependence are usually observed in single crystals and amorphous solids and with difficulty in polycrystalline solids (Mason, 1958). Equation (10) gives the frequency dependence of thermodynamic losses that arise when longitudinal waves produce heat flow from dilatation to compression regions. 
Viscous losses (Auld, 1973) also exhibit a second-power frequency dependence but are generally negligible in solids. Models for absorption losses caused by dislocation vibrations, relaxation effects, and internal friction predict second-power frequency dependence down to frequency independence (Granato and Lücke, 1956; Seeger and Schiller, 1962; Truel1, et al., 1969). Absorption losses due to magnetic domains, electrons, or phonons are special cases involving ferromagnetic materials, very high frequencies, or cryogenic temperatures, respectively, and are not considered here.

\section{Combined Expressions}

Total attenuation coefficients are usually written ad hoc as sums of coefficients for scattering and absorption. For example, equations (7) and (9) are combined to form an expression for the total attenuation coefficient $\alpha$, where

$$
\alpha_{h p}=c_{h} f+c_{p} a f^{2}
$$

or equations (6) and (9) are combined to get

$$
\alpha_{h r}=c_{h} f+c_{r} a^{3} f^{4}
$$

Combined expressions as in equations (11) and (12) are convenient for fitting experimental data and for analyzing the contributions of attenuation mecha-nisms and underlying microstructural factors.

An inconsistency occurs in attempting to combine the expressions for Rayleigh and stochastic attenuation in equations (6) and (7) because as ultrasonic frequency increases, the fourth-power term for Rayleigh scattering becomes dominant. This contradicts the expectation that for higher frequencies, as wavelength diminishes and approaches grain size, the second power of frequency due to stochastic scattering should dominate. Papadakis (1965b) and others have dealt with this issue by applying equations (6) and (7) piecemeal and adapting a smoothing function to form continuity between the Rayleigh and stochastic regimes. Smith (1984) has observed that the transition from Rayleigh to stochastic scattering depends on grain-size distribution and suggests introducing a probability function in scatter attenuation equations proposed by Roney (1950) and Serabian and Williams (1978).

Maisel (1984) overcomes the problem of expressing the transition from Rayleigh to stochastic scattering by using an electrical network analog to model ultrasonic scattering. Maisel's results suggest expressions for the attenuation coefficient that combine Rayleigh and stochastic frequency dependencies in a continuous function. One such expression is

$$
\alpha_{r p}=c_{r p} \frac{4 a^{3} k^{4}}{1+4 a^{2} k^{2}}
$$

where $c$ is a material constant incorporating average fractional deviations of density and compressional modulus, a is mean scatterer size, $k$ is wave number $(2 \pi f / v)$, and $v$ is velocity. The formulation of equation (13) allows 
the fourth power of frequency to dominate at low frequencies and the second power to dominate at high frequencies. A similar formulation was derived by Perkeris (1947) by assuming an autocorrelation function for polycrystalline inhomogeneity. Although both formulations allow a smooth transition from fourth- to second-power frequency dependence with increasing ultrasonic frequencies, no allowance is made for frequency independence at extreme frequencies in accordance with equation (8). Rokhlin (1972) describes a generalized formulation that encompasses Rayleigh, stochastic, and diffusion scattering. However, in Rokhlin's approach a smooth transition between scattering regimes is lost because of the periodic nature of the sine function that was used.

\section{MICROSTRUCTURE CONSIDERATIONS}

\section{Grain Size}

Before dealing with transfer function synthesis we briefly review some relevant aspects of the interplay of microstructure and the previously discussed formulations for ultrasonic attenuation.

A universally cited quantity for characterizing polycrystalline microstructures is "mean grain size." This quantity is used regardless of difficulties inherent in measuring it or assigning appropriate values to it, especially in materials that exhibit complex microstructures (subgrains, second phases, etc.). Nevertheless it is appropriate, for many single-phase polycrystallines with "uniform" microstructures, to speak of a mean grain size. This depends on discovering a definitive grain-size distribution function (DeHoff, 1968). Most polycrystallines exhibit grain-size distributions that are highly skewed and nonGaussian because of a high incidence of large grains (Papadakis, 1964b, 1965b; Schückler, 1968; Evans, et al. 1978; Smith, 1984; Tittmann, et al., 1985).

There are polycrystalline aggregates that have microstructures with readily defined mean grain sizes (Schuickler, 1968). When expressions of the form of equations (11) and (12) are fitted to experimental attenuation data for samples of these materials, a new expression for the same sample after annealing to a different mean grain size can be obtained by modifying the coefficients of frequency by a numerical scale factor (e.g., the ratio of mean grain sizes). This has been demonstrated for polycrystalline samples of nickel and copper where measurements were predominantly in the stochastic scattering regime (i.e., principal wavelengths commensurate with grain size (Generazio, 1985)). A general scaling parameter for grain size has been described by Serabian and Williams (1978).

Scaling of attenuation expressions probably applies only to homomorphic microstructures having grain-size distribution functions that are invariant with changes in mean grain size. Photomicrographs of the successive heat treatments produced by Generazio (1985) seem to scale (i.e., map) exactly in this sense. Apparently scaling can be done even with rather complex microstructures despite difficulties in producing or identifying homomorphic grainboundary patterns. 
Scattering theories usually consider ensembles of scattering centers embedded in a featureless continuum. Current theories for polycrystallines account only for scattering by equiaxed grains and neglect effects of texture, anisotropy, grain-size variations, etc. Indeed, well-formulated theories for attenuation exist only for the simple case of single-phase polycrystalline solids with virtually identical, equiaxed grains and for frequencies that satisfy the conditions $k a \ll 1$ or $k a \gg 1$, where $k$ is wave number and a is mean grain (scatterer) size.

Underlying equations (6) and (7) is the assumption of single scattering by independent scatters; the conceptual and theoretical difficulties that arise with multiple scattering where scatterers interact and velocity dispersion effects are likely to arise are ignored. For example, equation (6) is based on the "Rayleigh criterion" and ideal elastic scatterers that are sufficiently separated to ensure single scattering (Truell, et al., 1969).

Fourth- and second-power relations given in equations (6) and (7), respectively, have been experimentally confirmed for special cases (Winkler and Murphy, 1984). Papadakis (1981) and Sayers (1985) realized the fourthpower relation of equation ( 6 ) by measuring scattering due only to "minority" phases (e.g., carbon nodules and inclusions) in polycrystalline aggregates. Evans, et al., (1978) used a similar approach for materials with sparse distributions of "predominant" scatterers in a polycrystalline "continuum."

Apparently, when used with materials exhibiting discrete, widely separated, "strong" scattering centers, the Rayleigh criterion is satisfied and the difficulties due to multiple scattering are avoided. It seems remarkable that frequency dependences predicted by rather simplified scattering models can be realized even with complex polycrystallines.

\section{Empirical Correlations}

For most polycrystallines the overall experimentally determined frequency dependence of attenuation is not an integral power. Exponents on frequency even in the Rayleigh or stochastic regimes rarely equal exactly 4 or 2 , respectively (Papadakis, 1981). This is probably due partly to imprecisions in experimental measurements and to attenuation data being defined only within the bandwidth used to interrogate the sample. Within limited bandwidths the best $f$ it of experimental data simply reflects only a portion of the actual attenuation function.

Because of the aforementioned factors it is often possible to fit attenuation data with expressions that allow the exponent on frequency to be an experimentally determined variable (Vary, 1980a,b). Among the simplest expressions of this kind is

$$
>a<=\operatorname{cf}^{\mathrm{m}} \quad \mathrm{f}_{1}<\mathrm{f}<\mathrm{f}_{2}
$$

where $>\alpha<$ denotes that $\alpha$ is empirically defined only in a limited frequency range and where $m$ may assume noninteger values. The quantities $c$ and $m$ will change correspondingly with microstructural changes that affect 
attenuation (mean grain size, grain size distribution, etc.). Experimental results show that equation (14) is appropriate for fitting attenuation data over a frequency range that spans the Rayleigh scattering regime for a variety of polycrystalline materials (Papadakis, 1964a, 1976; Vary, 1978; BozorgGrayeli, 1981; Vary and Hu11, 1982, 1983). It will be seen that equation (14) gives statistically valid descriptions of data in the Rayleigh regime.

\section{Grain Interfaces}

From a purely physical standpoint grain facets and surface areas should influence mechanical properties of polycrystallines. This is certainly true of properties that depend on the surface energy of grains, properties affected by the grain-boundary thickness, properties for which grain boundaries are obstacles, and properties connected with grain- and phase-boundary migrations and obliterations. The transfer function concept, described next, provides a basis for a unified expression for attenuation that incorporates the previously discussed attenuation mechanisms and also a means for studying the effects of variations in grain-boundary energy transmission factors.

\section{TRANSFER FUNCTION SYNTHESIS}

\section{Approach}

An attenuation transfer function for polycrystalline solids is defined in terms of a lattice model that incorporates the effects of grain-boundary (interface) spacing and energy transmission across interfaces. The lattice model is generalized by the introduction of probability distribution functions to account for variations in grain and scatterer size and boundary spacing. The transfer function concept provides a convenient and rational basis for incorporating the previously described attenuation mechanisms into a unified expression for ultrasonic attenuation.

\section{Interface Transfer Function}

The transfer function of an interface between two media is defined in terms of the reflection coefficient at the interface. We assume ideal flat interfaces between dispersion-free media with unequal acoustic impedances. In this case the reflection coefficient $R$ is taken as a frequency-independent Heaviside step function in the frequency domain, corresponding to a delta (impulse) function in the time domain, see figure 3 and appendix $B$.

The reflection coefficient $R$ in equations (1) to (4) conforms with the interface transfer function notion given here. If it becomes necessary to consider velocity dispersion or nonideal (thick, strained, acoustically rough, etc.) interfaces, expressions for $R$ (and the quantity $G$, defined below) would simply assume frequency-dependent forms (Papadakis, 1976; Kinsler, et al., 1982; Generazio, 1984). 
The lattice model illustrated in figure 4 represents a volume that consists of series of plane parallel interfaces separating thin layers with unequal acoustic impedances (compare with Rokhlin (1972), where a volume is divided into equal rectangular parallepipeds). The model is one dimensional and accounts for energy losses suffered by longitudinal rays traveling at normal incidence to the interfaces. Each of the $n$ internal interfaces has a transfer function defined in terms of an internal reflection coefficient $G$ (appendix B). For the moment consider each layer as a featureless (scatterer free) medium with an intrinsic attenuation function

$$
A=|A(f)|=\exp \left(-\alpha_{i} \ell\right)
$$

Where $\ell$ is layer thickness and $A$ is the magnitude (power) spectrum of $A(f)$. As developed in appendix $C$ the transfer function $T$ for the lattice model can be reduced to the form

$$
T=(H-G)^{2 n} A^{2(n+1)}\left[\sum \ldots\right]
$$

where $H=|H(f)|=1$ is the Heaviside unit step function that will be rendered as 1 hereinafter (i.e., $(H-G)=(1-G)$ ). The multiple convolution (multiple reflection) factor $\left[\sum \ldots\right]$ in equation (16) is

$\left[\sum \ldots\right]=\left[\sum_{1}^{q}(R G)^{q-1} A^{2(q-1)}\right]^{2}\left[\sum_{1}^{q} G^{q-1} A^{2(q-1)}\right]\left[\sum_{1}^{q} G^{2(q-1)} A^{2(q-1)}\right]^{2(n-1)}$

The number of first-order multiple reflections in each lattice layer is $q-1$, and the number of layers is $n+1$. Equation (16) defines $T$ in terms of power (energy) reflection functions $R$ and $G$ (appendix $A$ ). The terms $A, G, R$, and $T$ are Fourier transforms. The various powers and products of $A, G$, and $R$ are convolutions of their time-domain counterparts. For example, RG in equation (17) is the convolution of corresponding time-domain quantities. The quantity $\left[\sum \ldots\right]$ given in equation (17) explicitly contains only first-order multiple reflections. It will be seen that this is sufficient for generalizing the model.

\section{Lattice Model with Scattering}

The lattice model may be extended to include scattering by taking $A$ in equations (16) and (17) to consist of both intrinsic and extrinsic attenuation factors,

$$
A=\left[\exp \left(-\alpha_{i} \ell\right)\right]\left[\exp \left(-\alpha_{e} \ell\right)\right]
$$

If it is postulated that each layer contains an independent scattering center in addition to intrinsic attenuation, equation (16) takes the form

$$
T=(1-G)^{2 n}\left[\exp \left(-\alpha_{i} l\right) \exp \left(-\alpha_{e} l\right)\right]^{2(n+1)}\left[\sum \ldots\right]
$$


Let the intrinsic attenuation be due to anelastic hysteresis, and in accordance with Nowick and Berry (1972) express it as

$$
\alpha_{i}=c_{h} f=\left(\frac{h}{v}\right) f
$$

where $h$ is a hysteresis constant and $v$ is velocity. For extrinsic attenuation take the Rayleigh scattering expression derived by Mason and McSkimmin (1947),

$$
\alpha_{e}=c r^{a^{3} f^{4}}=\left(\frac{16 \pi^{4} K}{3 v^{4}}\right) a^{3} f^{4}
$$

where $a$ is mean scatterer size (with $4 \pi a^{3} / 3$ replacing the mean grain volume in the Mason and McSkimmin (1947) derivation) and $k$ is elastic anisotropy ( $i . e .$, average change in elasticity encountered from one layer to the next (Mason and McSkimmin, 1948; Lifsitz and Parkomovskii, 1950; Merkulov, 1957)).

\section{Generalization for Polycrystallines}

We generalize the lattice model to resemble a polycrystalline by postulating unequal layer thicknesses and nonparallel interfaces. Thus any ray traveling at normal incidence to the front and back surfaces (still mutually parallel) encounters a sequence of interfaces similar to those in a polycrystalline aggregate.

Combining equations (4) and (19), noting that, for large values of $n$, $(n / x)=(1 / \ell)$ while $(n+1) \ell=x$ and taking $\ell$ as mean layer thickness, an expression is obtained for $\alpha$ in terms of $T$,

$$
\alpha=-\left(\frac{1}{l}\right) \ln (1-G)+\alpha_{i}+\alpha_{e}+\left(\frac{1}{2 x}\right) \ln \left[\sum \ldots\right]
$$

The quantity $G$ is now taken as average reflection coefficient for the randomly oriented interfaces. If $G$ is sufficiently small and energy is indeed scattered away in lattice layers, the contribution of higher order terms in the sumnations in equation (17) may be ignored. This is equivalent to setting $q=1$ in equation (17) so that $\ln [\Sigma \ldots]=0$ (see DISCUSSION). Although the energy transmission coefficient $I-G$ may approach unity, the interface transparency term ln $(1-G)$ is always finite (i.e., a nonzero negative).

Given the preceding argument and noting that $l$ corresponds to a mean grain size represented by the quantity a and combining equations (20) to (22),

$$
\alpha=-\left(\frac{1}{a}\right) \ln (1-G)+\left(\frac{h}{v}\right) f+\left(\frac{16 \pi^{4} K}{3 v^{4}}\right) a s^{2} f^{4}
$$

Although a spherical scatterer with volume $4 \pi \mathrm{a}^{3} / 3$ is implicit to equation (21), in equation (23) scatterer geometry is made abstract so that $s$ is an effective mean scatterer diameter while a is mean scatterer length. Thus 
we allow for nonequiaxed grains or scatterers with aspect ratios a/s of 1 or greater. Rokhlin (1972) assumes grains to be parallelepipeds having square ends but different side lengths in the direction of wave propagation.

\section{Generalization for Grain-Size Distribution}

The range of grain sizes usually contained in a polycrystalline can be described by a histogram or, for a large grain population, by an analytical probability distribution function. Grain-size distributions are usually nonGaussian and their probability distributions are typically log-normal functions (DeHoff, 1968; Schuickler, 1968; Smith, 1984). A function that describes grain-size distributions representative of most polycrystalline solids is given by

$$
p(y)=\left(\frac{4 y}{a}\right) \exp \left(\frac{-2 y}{a}\right) \quad p(y)=0 \text { for } y<0
$$

where $y$ is a spatial (length) variable and $a$ is mean grain size. We have pointed out that the quantity $\alpha$ is defined in the frequency domain and can therefore be treated as the Fourier transform of a time-domain quantity. To incorporate probability distribution functions in the expression for $\alpha$ given by equation (23), we transpose to the tine domain, take the Fourier transform, and find the results of convolution with probabalistic factors in equation (23). Therefore let

$$
p(t)=\left(\frac{4 t}{u^{2}}\right) \exp \left(\frac{-2 t}{u}\right) \quad p(t)=0 \text { for } y<0
$$

where $u$ is mean transit (grain delay) time corresponding to mean grain size $a$ and $a=u v$, where $v$ is average velocity (fig. 5). The Fourier transform of $p(t)$ is

$$
P(f)=\left[1+(\pi u f)^{2}\right]^{-1} \exp \left[-2 i \tan ^{-1}(\pi u f)\right]
$$

The phase spectrum given by the exponential term in equation (26) is ignored since we are interested only in the magnitude spectrum of $P(f)$ (i.e., $P=|P(f)|)$. The implicit assumption is that phase interference effects are insignificant. In general, the probability distribution function of the scatterer diameter $s$ is not identical to that of its axial length $a$. Therefore we admit two separate expressions for $P$,

$$
\begin{aligned}
& P_{a}=\left[1+\left(\pi u_{a} f\right)^{2}\right]^{-1} \\
& P_{s}=\left[1+\left(\pi u_{s} f\right)^{2}\right]^{-1}
\end{aligned}
$$

where subscripts $s$ and a refer to mean scatterer diameter and length, respectively. That is, $u_{a}=a / v$ and $u_{s}=s / v$. 
Now consider the first term in equation (23) as a probabilistic quantity. In the frequency domain the probabilistic weighting of the reflection function for transit time variations is GP and the probabilistic weighting for trans-mission is $G(1-P)$. Therefore the frequency dependence of the boundary energy transmission term $1-G$ in equation $(23)$ is $\ln [1-G(1-P)]$. The expression for $P$ in equation (27) will be used for this case. The middle term in equation (23) is independent of grain size and remains unaltered. In the third term of equation (23) a and $s$ are probabilistic so that

$$
\alpha=-\left(\frac{1}{v u_{a}}\right) \ln \left[1-G\left(1-P_{a}\right)\right]+\left(\frac{h}{v}\right) f+\left(\frac{16 \pi^{4} K}{3 v u_{a}}\right) u_{a}^{2} u^{2} f^{4} P_{a} P^{p}
$$

where $u_{a}=a / v, u_{s}=s / v$, and $v$ is average velocity. The corresponding probability functions $P_{2}$ and $P_{S}$ are to be defined according to the nature of the various microstructural factors that govern attenuation (grains, precipitates, etc.) in a particular solid. A further generalization would take grain-boundary spacing and scatterer spacing (and dimensions) to be independent of each other.

\section{VERIFICATION AND APPLICATIONS OF MODEL \\ Comparison with Scattering Theory}

We examine a special case of equation (29) wherein grains are equiaxed scatterers (i.e., $a=s=v u$ ). Also, take $P_{a}=P_{s}=P=|P(f)|$. For the moment ignore the first two terms in equation (29) so that consequences of the preceding derivations may be compared with classic expressions for scattering. The extrinsic attenuation (third) term of equation (29) expands to

$$
\alpha_{e}=\left(\frac{16 \pi^{4} k}{3 v u}\right)\left\{\frac{(u f)^{4}}{\left[1+(\pi u f)^{2}\right]^{2}}\right\}
$$

For $\lambda \gg \pi$ equation (30) reduces to the expression for Rayleigh scattering (Mason and McSkimmin, 1947),

$$
\alpha_{\text {er }}=\left(\frac{16 \pi^{4} K}{3 v^{4}}\right) a^{3} f^{4}
$$

For $\lambda \cong \pi a$ equation (30) reduces to the expression for stochastic (phase) scattering,

$$
\alpha_{e p}=\left(\frac{16 \pi^{2} K}{3 v^{2}}\right) a f^{2}
$$

For $\lambda \ll \pi a$ equation (30) reduces to the expression for diffusion scattering,

$$
\alpha_{\text {ed }}=\left(\frac{16 \mathrm{~K}}{3}\right) \mathrm{a}^{-1}
$$


Thus we have in equation (30) an expression for extrinsic attenuation that covers all theoretically postulated scattering mechanisms continuously over all frequencies.

Although we have adopted the scatter attenuation expression of Mason and McSkimmin (1947), the form developed by Lifsitz and Parkomovskii (1950) would be appropriate for defining extrinsic attenuation in equations (21) and (30) to account for elastic randomization and attendant effects of mode conversion. However, it is convenient and sufficient for our purposes to continue using the expression for extrinsic attenuation given in equation (21).

\section{Comparison with Experiment}

Consider a simple polycrystalline where, as in the previous section, grains and other scatterers are taken to be identical, so that $a=s=v u$, where $a$ is mean grain size, $u$ is mean grain delay, and $v$ is mean velocity. Using equation (30) for extrinsic scattering, equation (29) becomes

$$
\alpha=-\left(\frac{1}{a}\right) \ln \left(1-G\left\{1-\left[1+(\pi u f)^{2}\right]^{-1}\right\}\right)+\left(\frac{h}{v}\right) f+\left(\frac{16 K}{3 a}\right)\left\{\frac{(\pi u f)^{4}}{\left[1+(\pi u f)^{2}\right]^{2}}\right\}
$$

We assign appropriate values to $G, K$, and $h$ for comparing attenuation coefficients predicted by equation (34) with experimental data for nickel, copper, and iron polycrystallines.

Serabian and Williams (1978) estimated hysteresis constants for several metals (e.g., $\mathrm{h}=2 \times 10^{-5}$ for 97.4 percent iron alloys and $\mathrm{h}=3 \times 10^{-4}$ for 98.9 percent nickel alloys). Herein we assume $h=3 \times 10^{-4}$ for 99.99 percent copper.

Both $G$ and $K$ are estimated from equivalent equations and may be taken to be identical for cubic crystals (Mason and McSkimmin, 1947, 1948; Mason, 1958):

$$
K=\left[\frac{\left\langle c_{11}-\left\langle c_{11}\right\rangle\right\rangle}{\left\langle c_{11}\right\rangle}\right]^{2}=\left(\frac{z_{2}-z_{1}}{z_{2}+z_{1}}\right)^{2}=G
$$

where $c^{\prime}$ is the elastic tensor coefficient (compression modulus) and $\left\langle c^{\prime}\right\rangle$ is its average value. The $z=\rho v$ terms are defined in appendix $A$ and are based on principal longitudinal velocities. We can assume that for any given material $G$ is directly proportional to $\mathrm{K}$ and that for cubic crystallites $\mathrm{G}=\mathrm{K}$. According to Mason and McSkimmin (1948) $\mathrm{G}=\mathrm{K}=0.0074$ for copper, 0.0067 for iron, and 0.0072 for nickel.

Attenuation-versus-frequency curves generated with equation (34) are compared with experimental curves for the cubic materials iron, copper, and nickel in figures 6 to 8 . The estimated values for $K$ and $G$ do not account for the effects of oblique incidence or mode conversion at boundaries; these effects would increase their effective values. Therefore the values for $k$ cited above should give low predictions of attenuation since shear waves produced by mode conversions are more effectively scattered. Elastic anisotropy 
for shear waves can exceed that for longitudinal waves by perhaps a factor of 10. It is probably for this reason that the best agreement with experimental data is obtained when we double the estimated values for $K$ for use in equation (34). It will be seen that $K$ is in general a composite quantity containing other scattering factors.

Figure 6 compares original experimental data of Merkulov (1957) for iron with attenuation-coefficient-versus-frequency curves generated with equation (34). Excellent agreement between the experimental and predicted attenuation coefficients is evident in figures $6(a)$ and (b) for the particular values selected for $K, G$, and $h$ for iron, even to the extent that curve crossovers at approximately $14 \mathrm{MHz}$ are predicted. Figures $6(\mathrm{c})$ and (d) exhibit attenuation curves for iron over a broader frequency range of $100 \mathrm{MHz}$, as opposed to the $20-\mathrm{MHz}$ range in figure $6(\mathrm{~b})$. Figure $6(\mathrm{c})$ is based on $K=0.0067$ and figure $6(d)$ on $K=0.0134$ in equation (34) for grain sizes from 40 to $250 \mu \mathrm{m}$. Figures 7 and 8 compare original experimental data of Generazio (1985) for copper and nickel, respectively, with attenuation-versus-frequency curves generated with equation (34).

\section{Data simulation for Curve Fitting}

It is apparent that prior knowledge of the material parameters $h, G$, and $K$ is needed to predict the attenuation coefficient with equation ( 34 ). Estimates of these quantities were sufficient to simulate attenuation curves for iron, copper, and nickel in figures 6 to 8 . We illustrate an empiricalgraphical approach for estimating and verifying values for $h, G$, and $K$ in solids, where these quantities are not easy to determine.

Our approach is founded on the observation that over a limited frequency range attenuation data can of ten be fitted quite well with equation (14) (Papadakis, 1964a; Vary, 1978, 1982; Bozorg-Grayeli, 1981). It has been shown that for data in the Rayleigh regime extrapolations based on the empirical constants $c$ and $m$ lead to excellent correlations with mechanical properti.es (e.g., fracture toughness)(Vary, 1978; 1979; Vary and Hull, 1982, 1983).

No analytical proof is offered to show why an equation of the form of equation (14) should be expected to represent either equation (34) or (29) in any particular frequency regime. However, we will show that equation (14) satisfactorily represents experimental data in restricted frequency regimes by using equation (34) to generate raw data sets in the Rayleigh regime. The point is illustrated by simulating data sets for a range of mean grain size $a$ and values of $K, G, h$, and $v$ that represent iron.

A series of discrete data sets generated with equation (34) are exhibited in figure 9 for the frequency range 10 to $100 \mathrm{MHz}$, where the Rayleigh wavelength criterion is satisfied (i.e., $\lambda \gg \pi$ ). We ignored data below $15 \mathrm{MHz}$, where diffraction effects are most pronounced for actual data sets. Treating the simulated data as actual raw attenuation data, we determined $c$ and $m$ values by a least-squares fit to equation (14) for each "material sample." The $c$ and $m$ values and the correlation coefficients of the curve fittings were tabulated for each data set. As seen in figure 9 calculated frequency exponents $m$ were clearly nonintegral and ranged from approximately 1.8 to 2.3 , corresponding to mean grain sizes from 2 to $20 \mu \mathrm{m}$, in the case illustrated. The simulated exponents spanned a range similar to experimentally 
determined frequency exponents for a number of polycrystallines in the Rayleigh regime (Vary, 1978).

F'or the material parameters chosen for generating data for figure 9, the correlation coefficient was always greater than 0.999 . Thus by treating the simulated data precisely the way one could treat a set of actual attenuation data, we found that equation (14) renders a statistically valid representation within the frequency bounds specified.

\section{Interrelation of Parameters}

In addition to representing attenuation data for a given material sample within specified frequency bounds, the parameters $c$ and $m$ are interrelated for variously conditioned samples of the same material. As an example, exper-imentally determined values of $c$ and $m$ for two maraging steels were plotted and tabulated ( $\mathrm{fig} .10$ ). All materials for which the quantities $c$ and $m$ have been experimentally measured exhibit the strong functional relation between $c$ and $m$ evident in figure 10. The quantities $c$ and $m$ are evidently interdependent for any set of related materials or samples of the same material that apparently differ due to metamorphic changes brought about by heat treatment or other thermomechanical processing methods that retain global microstructural patterns but alter mechanical properties.

By varying the material parameters $G, K, h$, and $v$ in equation (34) and allowing the mean grain size to take representative values, the $c$ and $m$ quantities corresponding to any particular material can be duplicated. Parametric fields based on varying $G, K$, and $h$ appear in figure 11 . The fields in figure 11 were created by using the curve-fitting method illustrated in figure 9 (i.e., by simulating attenuation data with equation (34), fitting with equation (14) in the 15 - to $100-\mathrm{MHz}$ range and including in the fields only points for which the curve fit correlation exceeded 0.999). Values selected for $h, G$, and $K$ corresponded to those for maraging steel and $v$ was taken to equal $0.56 \mathrm{~cm} / \mu \mathrm{s}$, the mean value for a 250 -grade maraging steel (Vary, 1978).

Coplotted in each field in figure 11 are data for the 250-grade maraging steel from figure 10 for which the mean grain size ranged from about 8.5 to $13 \mu \mathrm{m}$. Accordingly, in figure 11(a) the maraging steel data fall near the curve for mean grain size a equal to $10 \mu \mathrm{m}$. This is not fortuitous, since the location and shape of the field, relative to experimental $c$ and $m$ values for the maraging steel, depend on judicious selection of values for $h$ and G. Figures $11(\mathrm{~b})$ and (c), where we assume that $a=10 \mu \mathrm{m}$, appear to confirm the values $G=0.007$ and $h=0.00002$ for the $250-$ grade maraging steel.

An unexpected result is that each field in figure 11 indicates that the quantity $K$, the elastic anisotropy factor, varies by approximately a half order of magnitude among the maraging steel samples represented. Similar results were obtained for the 200-grade maraging steel data in figure 10 . This supports the notion that a material will have a different value of $k$ after thermomechanical conditioning. Indeed, it has been suggested that $k$ should be viewed as a composite quantity that includes a scattering coefficient (Mason, 1958; Papadakis, 1965a; Serabian and Williams, 1978). Heat treatment, strain, deformation, etc., alter attenuation properties due to effects on 
dislocations and grain boundaries (Serabian, 1985). These effects are apparent when experimental values for $c$ and $m$ are coplotted in appropriate parametric fields as in figure 11.

\section{DISCUSSION}

General

Our goal has been to develop a unified, comprehensive expression for ultrasonic attenuation. The incentive was to $f$ ind a rational basis for identifying and evaluating key microstructural factors that govern mechanical properties. We noticed that although previous expressions for attenuation are theoretically self-consistent, they provide only piecemeal, fragmented descriptions of ultrasonic attenuation mechanisms over the broad frequency range relevant to materials characterization.

We describe a transfer function concept and a heuristic lattice model that form bases for incorporating well-known attenuation mechanisms into a unified expression for ultrasonic attenuation. Although we ignore phase interference effects, our generalized analytical expression continuously accounts for the waxing and waning of all of the attenuation mechanisms previously embodied in separate equations. The generalized equation is shown to predict salient features of experimental attenuation-versus-frequency curves obtained for typical polycrystalline solids.

We believe that our chief contribution is the transfer function approach, in which expressions for attenuation coefficients are recognized and handled as frequency-domain transforms of time-domain quantities.

\section{Microstructure Encompassed}

We are concerned with polycrystallines, where ultrasonic attenuation depends primarily on such extrinsic microstructural factors as grain-size distribution and properties of grain interfaces. Using equation (34) we concentrate on relatively simple fine-grained, isotropic, homogeneous materials with mean grain sizes in the range $1 \mu \mathrm{m}$ to $>100 \mu \mathrm{m}$. We expect that the concepts given herein apply equally well to anisotropic materials and to amorphous or single-crystal materials that exhibit microporosity, dispersoids, etc. For example, equation (29) admits additional variables to account for grain anisotropy and bimodal distribution functions for dispersed scatterers.

\section{Probability Distribution Functions}

It should be apparent that our selection of a probability distribution function for use in equation (29) and equations developed from it was quite deliberate. In addition to representing typical polycrystalline grain populations, the probability distribution function given in equation (25) has salutary mathematical properties. These are evident in the consequences exhibited by equations (31) to (33), which depend on the form of the probability function. Moreover, once the mean is specified for the distribution function in equation (25), the variance is specified also (i.e., the variance equals half the square of the mean). Alternative distribution functions, such as Gaussians 
or combinations of normal and log-normal, may provide better descriptions of particular grain populations. Our immediate purpose was to indicate a rational method for introducing probabilistic factors in attenuation equations.

\section{Interface Transfer Function}

We applied the transfer function concept to a generalized version of the lattice model to represent polycrystalline materials. One consequence of the model was the introduction of a grain-boundary (interface) energy transmission function (e.g., represented by the term (1/a) ln ( $1-\mathrm{G}$ ) in eq. (23)). This term is similar to the diffusion scattering expressions of equations (8) and (33). It is related to $\mathrm{K}$, the elastic anisotropy factor in equation (33). Unlike the extrinsic (scatter) attenuation terms that include $\mathrm{K}$, the In $(1-G)$ term does not vanish in the absence of scatterers. Our model requires (and retains) it to account for the energy lost by reflection at internal interfaces. In general, $G$ is frequency dependent (as in the case of nonideal, thick, or acoustically rough interfaces, see appendix $A$ ).

\section{Multiple Reflection Term}

In our generalization the multiple reflection term [ $\left.\sum \ldots\right]$ of equation (17) is ignored. This is justifiable if the model represents polycrystallines that exhibit no significant forward-or backscatter. The waveform shown in figure 1 is a typical of this case (i.e., where no evidence of scatter wavelets appears either between or near the main echoess). This would not be true for coarse-grained materials that can exhibit considerable backscatter. In general, the $\left[\sum \ldots ..\right]$ term needs to be considered (e.g., when first- and second-order multiple reflections are significant as in layered structures (composite laminates)).

The quantity $\left[\sum \ldots\right]$ collects all multiple reflections that are not attenuated or deflected out of the interrogating beam. Referring to equations (17) and (22), we notice that, in our model, as $G \rightarrow 0$ the lattice interfaces become transparent, and overall attenuation will be due to intrinsic absorption and extrinsic scattering, say, due to point scatterers, voids, etc. In this case equation (22) reduces to $\alpha=\alpha_{i}+\alpha_{e}$. Note that for all values of $G$ the first term of each summation in equation (17) equals 1 .

When the average internal reflection coefficient $G$ lies between 0 and 1 , the interfaces assume varying degrees of opacity. For sufficiently large values of $G$ we can no longer ignore the first and higher power terms associated with $q=2,3, \ldots$, in equation (17). In this latter case backscatter would become significant and the effects of the reflection coefficient $R$ at the transducer-specimen interface enter the expression for $\alpha$, as for example in equation (22). Under this condition as in the case of equation (4) measurement of $\alpha$ depends on apriori knowledge or independent measurement of $R$.

\section{Power Functions of Frequency}

Integral exponents on frequency, as given by equations (6) to (10), should not be expected to apply to experimentally determined relations between the attenuation coefficient and frequency. Our analysis and experimental evidence 
show that even over narrow frequency ranges attenuation is a nonintegral power function of frequency. Exponents on frequency will assume values between 1 and 4 according to the degree of influence of material parameters such as those appearing in equations (29) and (34). Indeed, figures 6 to 8 show that exponents are themselves functions of frequency.

Heretofore, we and other investigators have found it practical to use nonintegral, fixed exponents to describe empirical relations between attenuation and frequency within bounded regimes. By using the generalized attenuation equations we show that this approach is statistically valid (e.g., as in the case of $\mathrm{fig} .9$ ). However, it is now clear that considerably more information concerning microstructure may be extracted by applying the generalized expressions for attenuation in equations (29) and (34).

\section{Elastic Anisotropy and Related Factors}

Our preliminary analysis of the implications of the interrelation of the empirical quantities $c$ and $m$ from equation (14) confirmed the importance of considering variations in the effective values of $K$ (and $G$ ) in applying equation (34), especially to polycrystallines modified by thermomechanical processing. We infer that changes can occur in the effective values of $K$ that transcend changes in grain geometry (size, shape, etc.). These correspond to changes in mechanical properties (fracture toughness, yield strength, etc.) in materials in which only slight or no changes in grain size have occurred. In effect, equations (29) and (34) appear to provide entry to analysis of subtle metamorphic changes that arise from thermomechanical processing of polycrystallines.

\section{CONCLUDING REMARKS}

A unified expression for ultrasonic attenuation has been introduced to supplant currently diverse and fragmented equations. A transfer function concept and a heuristic lattice model with an interface energy transmission factor have been described as rational bases for combining a variety of attenuation mechanisms and probabilistic factors in a comprehensive equation. Although our model is essentially one dimensional and we ignore phase interference effects, the resultant generalized equation predicts salient features of experimental attenuation-versus-frequency curves obtained for typical polycrystalline solids. Some consequences of the unified expression have been explored relative to characterization of key microstructural factors that govern mechanical properties of polycrystallines. 


\section{APPENDIX A \\ REFLECTION COEFFICIENT AND ACOUSTIC IMPEDANCE}

The reflection coefficient can be defined either in terms of power (energy intensity) or amplitude (pressure) (Krautkrämer, 1969; Szilard, 1982). Taking $R$ as the reflection coefficient and $Q$ as the transmission coefficient across an interface, from medium 1 to 2 , we have for the power case,

$$
\begin{gathered}
R_{p}+Q_{p}=1 \\
R_{p}=\left(\frac{z_{2}-z_{1}}{z_{2}+z_{1}}\right)^{2} \\
Q_{p}=\frac{\left(4 z_{2} z_{1}\right)}{\left(z_{2}+z_{1}\right)^{2}}
\end{gathered}
$$

and for the amplitude case,

$$
\begin{aligned}
& R_{a}+1=Q_{a} \\
& R_{a}=\frac{z_{2}-z_{1}}{z_{2}+z_{1}} \\
& Q_{a}=\frac{2 z_{2}}{z_{2}+z_{1}}
\end{aligned}
$$

where $z_{1}$ and $z_{2}$ are acoustic impedances of media 1 and 2 ,

$$
\left.\begin{array}{l}
z_{1}=\rho_{1} v_{1} \\
z_{2}=\rho_{2} v_{2}
\end{array}\right\}
$$

$\rho$ is density, and $v$ is velocity.

In polycrystallines grain-.boundary interfaces will reflect as well as transmit ultrasonic energy because of acoustic impedance "jumps" across differently oriented contiguous crystals.

The preceding equations for reflection and transmission apply to ideal interfaces that have no thickness. If there is an intervening medium that has a finite thickness d such that d greatly exceeds the ultrasonic wavelengths transmitted, $d \gg \lambda$, the transmission and reflection coefficients are frequency dependent (Kinsler, et al., 1982). Letting $v_{3}$ be velocity in the intervening medium (medium 3 ) between media 1 and 2 and $z_{3}$ be its acoustic impedance, $R$ becomes a function of frequency $f$ with the magnitude 


$$
|R(f)|=\frac{\left(1-\frac{z_{1}}{z_{2}}\right)^{2} \cos ^{2}\left(\frac{2 \pi f d}{v_{3}}\right)+\left(\frac{z_{3}}{z_{2}}-\frac{z_{1}}{z_{3}}\right)^{2} \sin ^{2}\left(\frac{2 \pi f d}{v_{3}}\right)}{\left(1+\frac{z_{1}}{z_{2}}\right)^{2} \cos ^{2}\left(\frac{2 \pi f d}{v_{3}}\right)+\left(\frac{z_{3}}{z_{2}}+\frac{z_{1}}{z_{3}}\right)^{2} \sin ^{2}\left(\frac{2 \pi f d}{v_{3}}\right)}
$$

When $\lambda_{3}=\left(v_{3} / 2 \pi f\right) \gg d$, equation (A4) reduces to equation (AI). 


\section{APPENDIX B \\ REFLECTION COEFFICIENT TRANSFER FUNCTION}

In this paper the reflection coefficient $R$ is always taken as a frequency-domain quantity. For an ideal interface between two nondispersive media with unequal acoustic impedances, $R$ is independent of ultrasonic frequency (Papadakis, 1976; Generazio, 1984). We define $R$ as identical to the Heaviside step function $H(f)$, (Bracewell, 1978)( $f i g .3$ )

$$
|R(f)|=|H(f)|
$$

where

$$
|H(f)|=\left\{\begin{array}{lll}
1 & \text { for } & f \geq 0 \\
0 & \text { for } & f<0
\end{array}\right.
$$

The time-domain counterpart ( $i . e .$, inverse Fourier transform) of $R(f$ ) is then the delta (impulse) function $\delta$ defined by

$$
\int_{-\infty}^{+\infty} \delta(t) d t=1
$$

where $\delta=0$ for $t \neq 0$ and $t$ is time.

We adopt the additional formalism that the delta function is the inverse transform of $H(f)$ with arbitrary magnitude and phase spectra. This allows the delta function to be positioned at any point in the time domain and to have any value $w$ (Bendat and Piersol, 1980) so that

$$
\int_{t_{0}-\varepsilon}^{t_{0}+\varepsilon} w \delta\left(t-t_{0}\right) d t=w
$$

where

$$
w \delta\left(t-t_{0}\right)=\left\{\begin{array}{lll}
\infty & \text { for } & t=t_{0} \\
0 & \text { for } & t \neq t_{0}
\end{array}\right.
$$

and where $|H(0)|=w$ by definition of the Fourier transform of $\delta(t)$. In a nondispersive medium $|\mathrm{H}(\mathrm{f})|=\mathrm{w}$, a constant over all frequencies $f$ equal to or greater than 0 . 


\section{APPENDIX C}

\section{DEVELOPMENT OF LATTICE MODEL EQUATION}

A ray is traced through the lattice model as depicted in figure 4. starting with the "source" $E_{0}$, transmissions and first-order multiple reflections at each interface encountered are summed to determine $B_{1}$ and $B_{2}$.

The lattice front surface has the reflection coefficient $R$ as a result of coupling to a buffered transducer as in figure 1 . There are $n$ interfaces within the lattice, each with the identical reflection coefficient $G$. The back surface interfaced with a vacuum (or air) has a reflection coefficient of unity.

In this appendix, as through the main text, the quantities $A, B, G, E$, $F$, and $R$ are frequency-domain quantities (i.e., Fourier transforms of corresponding time-domain quantities). Reflection coefficients are based on the power definition given in appendix $A$ and therefore $B_{1}$ and $B_{2}$ must be taken as power (energy) spectra of corresponding waveforms.

Ignoring backscatter (second-order multiple reflections) caused by $E_{0}$, the first back echo is

$$
B_{1}=E_{0}(H-R)
$$

where $H$ is the Heaviside unit step function $H=|H(f)|=1$ (appendix $B$ ). The second back echo is

$$
\begin{aligned}
B_{2}=E_{0}(H-R) R(H-G)^{2 n} & {\left[\sum_{1}^{q}(R G)^{q-1} A^{2 q-1}\right]^{2} \cdot \cdot } \\
& {\left[\sum_{1}^{q} G^{q-1} A^{2 q}\right] \cdot \cdot \cdot\left[\sum_{1}^{q} G^{2(q-1)} A^{2 q-1}\right]^{2(n-1)} \cdot \cdots }
\end{aligned}
$$

where $A$ is unspecified intrinsic and/or extrinsic attenuation within the lattice layer and is identical in each layer. The echo $B_{2}$ is obtained by summing the internal transmissions and reflections $F_{1}, F_{2}, \ldots, F_{n}$ and $E_{1}$, $\mathrm{E}_{2}, \ldots, \mathrm{E}_{\mathrm{n}}$. For forward-going terms (e.g., $\mathrm{F}_{1}$ to $\mathrm{F}_{\mathrm{n}}$ )

$$
F_{1}=E_{0}(H-G) \sum_{1}^{q} R_{G}^{q_{G} q-1} A^{2 q-1}=F_{11}+F_{12}+\ldots+F_{1 q}
$$


where

$$
\begin{gathered}
F_{11}=E_{0} R(H-G) A \\
F_{12}=E_{0} R^{2} G(H-G) A^{3} \\
F_{13}=E_{0} R^{3} G^{2}(H-G) A^{5} \\
\cdot \\
\vdots \\
F_{1 q}=E_{0} R^{q_{G} q-1}(H-G) A^{2 q-1}
\end{gathered}
$$

and

$$
F_{n}=F_{(n-1)}(H-G) \sum_{1}^{q} G^{2(q-1)} A^{2 q-1}
$$

For returning echo terms (e.g., $E_{1}$ to $E_{n}$ )

$$
E_{1}=F_{n}(H-G) \sum_{1}^{q} G^{q-1} A^{2 q}=E_{11}+E_{12}+\ldots+E_{1 q}
$$

where

$$
\begin{gathered}
E_{11}=F_{n}(H-G) A^{2} \\
E_{12}=F_{n} G(H-G) A^{4} \\
E_{13}=F_{n} G^{2}(H-G) A^{6} \\
\vdots \\
E_{1 q}=F_{n} G^{q-1}(H-G) A^{2 q}
\end{gathered}
$$

and

$$
E_{n}=E_{(n-1)}(H-G) \sum_{1}^{q} G^{2(q-1)} A^{2 q-1}
$$

Then

$$
B_{2}=E_{n}(H-R) \sum_{1}^{q}(R G)^{q-1} A^{2 q-1}
$$




$$
\begin{aligned}
\frac{B_{2}}{R B_{1}}=(H-G)^{2 n_{A} 2(n+1)}\left[\sum_{I}^{q}(R G)^{q-1} A^{2(q-1)}\right]^{2} \cdot \cdot \\
\\
{\left[\sum_{I}^{q} G^{q-1} A^{2(q-1)}\right] \cdot \cdot \cdot\left[\sum_{1}^{q} G^{2(q-1)} A^{2(q-1)}\right]^{2(n-1)} \cdot \cdot \cdot }
\end{aligned}
$$

In equation (C10) ". . . indicates that the summations are to be multiplied together and that additional factors comprised of summations of second-order multiple reflections are omitted. These additional factors arise from backtransmissions and re-reflections that originate with first-order terms (e.g., $E_{1}, E_{2}, \ldots, E_{n}$ and $\left.F_{1}, F_{2}, \ldots, E_{n}\right)$.

Ignoring second-order multiple reflections within the lattice layers, the lattice transfer function $T$ is obtained by combining equation (C10) with equation ( 3 ) to obtain equation (16) in the main text. 
REFERENCES

Auld, B.A. (1973), Acoustic Fields and Waves in Solids, vol. 1. Wiley, New York.

Bhatia, A.B. (1967), Ultrasonic Absorption. Clarendon Press, Oxford, England.

Bendat, J.S, and Piersol, A.G. (1980), Engineering Applications of Correlation and Spectral Analysis. Wiley-Interscience, New York.

Bozorg-Grayeli, N. (1981), Acoustic Nondestructive Evaluation of Micro-

structure. Ph.D. Thesis, Stanford University, Stanford, CA.

Bracewell, R.N. (1978), The Fourier Transform and its Applications.

McGraw-Hill, New York.

DeHoff, R.T. (1968), The Statistical Background of Quantitative Metallography. Quantitative Microscopy, R.T. DeHoff and F.N. Rhines, eds., McGraw-Hill, New York, pp. 11-44.

Evans, A.G., et al. (1978), Ultrasonic Attenuation in Ceramics. J. Appl. Phys., vol. 49, no. 5, pp. 2669-2679.

Fitting, D.W. and Adler, L. (1981), Ultrasonic Spectral Analysis for Nondestructive Evaluation. Plenum Press, New York.

Generazio, E.R. (1985), The Role of the Reflection Coefficient in Precision Measurement of Ultrasonic Attenuation. Mater. Eval., vol. 43, no. 8, pp. 995-1004.

Generazio, E.R. (1985), Ultrasonic Verification of Microstructural Changes due to Heat Treatment. Analytical Ultrasonics in Materials Research and Testing, NASA CP-2383.

Goebbels, K. (1980), Structure Analysis by Scattered Ultrasonic Radiation. Research Techniques in Nondestructive Testing, Vol. 4, R.S. Sharpe, ed., Academic Press, London, pp. 87-157.

Granato, A. and Lücke, K. (1956), Theory of Mechanical Damping Due to Dislocations. J. Appl. Phys., vol. 27, no. 6, pp. 583-593.

Green, R.E., Jr. (1973a), Ultrasonic Investigation of Mechanical Properties. Treatise on Materials Science and Technology, Vol. 3, Academic Press, New York.

Green, R.E., Jr. (1973b), Ultrasonic Attenuation Detection of Fatigue Damage. Ultrasonics International 1973, IPC Science and Technology Press, Guilford, Surrey, England, pp. 187-193.

Green, R.E., Jr. (1981), Effect of Metallic Microstructure on Ultrasonic Attenuation. Nondestructive Evaluation: Microstructural Characterization, and Reliability Strategies, 0 . Buck and S.M. Wolf, eds., Metallurgical Society, AIME, Warrensdale, PA, pp. 115-132. 
Green, R.E., Jr. (1985), Ultrasonic and Acoustic Emmision Nondestructive Materials Characterization. Analytical Ultrasonics in Materials Research and Testing, NASA CP-2383.

Haines, N.F., Bel1, J.C., and McIntyre, P.J. (1978), The Application of Broadband U1trasonic Spectroscopy to the Study of Layered Media. J. Acoust. Soc. Am., vol. 64, no. 6, pp. 1645-1651.

Kinsler, L.E., et al. (1982), Fundamentals of Acoustics, 3rd ed., Wiley, New York, Chapter 6.

Kline, R.A. (1964), Measurement of Attenuation and Dispersion Using an Ultrasonic Spectroscopy Technique. J. Acoust. Soc. Am., vol. 76, no. 2, pp. 498-504.

Klinman, R., et al. (1980), Ultrasonic Prediction of Grain Size, Strength, and Toughness in Plain Carbon steel. Mater. Eval., vol. 38, no. 10, pp. 26-32.

Kolsky, H. (1963), Stress Waves in Solids. Dover, New York.

Krautkrämer, J. and Krautkrämer, H, (1969), U1trasonic Testing of Materials. Springer-Verlag, New York.

Lifsitz, I.M. and Parkhomovskii, G.D. (1950), Theory of Propagation of supersonic Waves in Polycrystals. Zhur. Ekspt1. Theo. Fiz., vol. 20, pp. 175-182.

Lïcke, K. (1956), U1trasonic Attenuation Caused by Thermoelastic Heat Flow. J. App 1. Phys., vol. 27, no. 12, pp. 1433-1438.

Maisel, J.E. (1.984), Preliminary Investigation of an Electrical Network Model for Ultrasonic Scattering. NASA CR-3770.

Mason, W.P. and McSkimin, H.J. (1947), Attenuation and Scattering of High Frequency Sound Waves in Metals and Glasses. J. Acoust. Soc. Am., vol. 19, no. $3, \mathrm{pp} .464-473$.

Mason, W.P. and McSkimin, H.J. (1948), Energy Losses of Sound Waves in Metals Due to Scattering and Diffusion. J. Appl. Phys., vol. 19, no. 10, pp. 940-946.

Merkulov, L.G. (1957), Investigation of Ultrasonic Scattering in Metals. Sov. Phys. Tech. Phys., vol. I, no. 1, pp. 59-69.

Nowick, A.S. and Berry, B.S. (1972), Anelastic Relaxation in Crystalline Solids. Academic Press, New York.

Papadakis, E.P. (1960), Ultrasonic Attenuation in S.A.E. 3140 and 4150 Steel. J. Acoust. Soc. Am., vol. 32, no. 12, pp. 1628-1639.

Papadakis, E.P. (1964a), Ultrasonic Attenuation and Velocity in Three Transformation Products in Steel. J. Appl. Phys, , vol. 35, no. 5, pp. 1474-1482.

Papadakis, E.P. (1964b), From Micrograph to Grain-Size Distribution With Ultrasonic Applications. J. Appl. Phys., vol. 35, no, 5, pp. 1586-1594. 
Papadakis, E.P. (1965a), Revised Grain Scattering Formulas and Tables.

J. Acoust. Soc. Am., vol, 37, no, 4, pp. 703-710.

Papadakis, E.P. (1965b), Ultrasonic Attenuation Caused by Scattering in Polycrystalline Metals. J. Acoust. Soc. Am., vol. 37, no. 4, pp. 711-717.

Papadakis, E.P. (1968), Buffer-Rod System for Ultrasonic Attenuation Measurements. J. Acoust. Soc. Am., vol. 44, no. 5, pp. 1437-1441.

Papadakis, E.P. (1970), Ultrasonic Attenuation and Velocity in SAE 52100 Steel Quenched From Various Temperatures. Metal1. Trans., vol. 1, no. 4, pp. 1053-1057.

Papadakis, E.P. (1975), Ultrasonic Diffraction From Single Apertures with Application to Pulse Measurements and Crystal Physics. Physical Acoustics: Principles and Methods, Vol. 11, W.P. Mason and R.N. Thurston, eds., Academic Press, New York, pp. 151-211.

Papadakis, E.P. (1976), Ultrasonic Velocity and Attenuation: Measurement Methods With Scientific and Industrial Applications. Physical Acoustics: Principles and Methods, Vo1. 11, W.P. Mason and R.N. Thurston, eds., Academic Press, New York, pp. 277-374.

Papadakis, E.P. (1981), Ultrasonic Attenuation Caused by Rayleigh Scattering by Graphite Nodules in Nodular Cast Iron. J. Acoust. Soc. Am., vo1. 70, no. 3, pp. 782-787.

Pekeris, C.L. (1947), Note on the Scattering of Radiation in an Inhomogeneous Medium. Phys. Rev., vol. 71, no. 4, pp. 268-269.

Reynolds, W.N. and Smith, R.L. (1984), Ultrasonic Wave Attenuation Spectra in Steels. J. Phys. D, vol. 17, no. 1, pp. 109-116.

Roklin, L.L. (1972), Scattering of Ultrasound in Polycrystalline Materials. Soviet Physics-Acoustics, vol. 18, no. 1, pp. 71-75.

Roney, R.K. (1950), The Influence of Metal Grain Structure on the Attenuation of an Ultrasonic Acoustic Wave. Ph.D. Thesis, California Institute of Technology, Pasadena, CA.

Sayers, C.M. (1985), Scattering of Ultrasound by Minority Phases in Polycrystalline Metals. Wave Motion, vol. 7, no. 1, pp. 95-104.

Schreiber, E., Anderson, O.L. and Soga, N. (1973), Elastic Constants and Their Measurement. McGraw-Hi.11, New York.

Schückler, F. (1968), [Determination of] Grain size [Distribution]. Quantitative Microscopy, R.T. DeHoff and F.N. Rhines, eds., McGraw-Hill, New York, pp. 201-265.

Scott, W.R. and Gordon, P.F. (1977), U1trasonic Spectrum Analysis for Nondestructive Testing of Layered Composite Materials. J. Acoust. Soc. Am., vol. 62, No. 1, pp. 108-116. 
Seeger, A. and Schiller, P. (1962), Bildung und Diffusion von Kinken als Grundprozess der Versetzungsbewegung bei der Messung der Inneren Reibung. Acta Metall., vol. 10, no. 4, pp. 348-357.

Serabian, S. and Williams, R.S. (1978), Experimental Determination of Ultrasonic Attenuation Characteristics Using the Roney Generalized Theory. Mater. Eval., vol. 36, no. 8, pp. 55-62.

Serabian, S. (1980), Frequency and Grain Size Dependency of Ultrasonic Attenuation in Polycrystalline Materials. Brit. J. Non-Destr. Test., vol. 22, no. 2 , pp. 69-77.

Serabian, S. (1985), Ultrasonic Material Property Determinations. Analytical Ultrasonics in Materials Research and Testing, NASA CP-2383.

Shukla, S.S. and Yun, S.S. (1981), Ultrasonic Attenuation in GaAs. J. Acoust. Soc. Am., vol. 70, no. 6, pp. 1713-1716.

Smith, R.L. (1982), The Effect of Grain Size Distribution on the Frequency Dependence of the Ultrasonic Attenuation in Polycrystalline Materials. Ultrasonics, vol. 20 , no. 5 , pp. 211-214.

Szilard, J. (1982), Ultrasonic Testing - Nonconventional Techniques, John Wiley, N.Y.

Tittman, B.R., Ahlberg, L.A., and Fertig, K. (1985), Ultrasonic Characterization of Microstructures in Powder Metal Alloy. Analytical Ultrasonics in Materials Research and Testing, NASA CP-2383.

Truel1, R., Elbaum, C., and Chick, B.B. (1969), Ultrasonic Methods in Solid state Physics. Academic Press, New York.

Vary, A. (1978), Correlations Among Ultrasonic Propagation Factors and Fracture Toughness Properties of Metallic Materials. Mater. Eval., vol. 36 , no. $7, \mathrm{pp} .55-64$.

Vary, A. (1979a), Correlations Between Ultrasonic and Fracture Toughness in Metaliic Materials. Fracture Mechanics, ASTM-STP-677, C.W. Smith, ed., ASTM, Philadelphia, PA, pp. 563-578.

Vary, A. (1979b), Computer signal Processing for Ultrasonic Attenuation and Velocity Measurements. Proceedings of the $12 \mathrm{th}$ symposium on Nondestructive Evaluation, W.W. Bradshaw, ed., Southwest Research Institute, San Antonio, TX, pp. $33-46$.

Vary, A. (1980a), Ultrasonic Measurement of Material Properties. Research Techniques in Nondestructive Testing, Vol. 4, R.S. Sharpe, ed., Academic Press, London, pp. 159-204.

Vary, A. (1980b), Concepts and Techniques for Ultrasonic Evaluation of Material Mechanical Properties. Mechanics of Nondestructive Testing, W.W. Stinchcomb, ed., Plenum, New York, pp. 123-141.

Vary, A. (1980c), Simulation of Transducer-Couplant Effects on Broadband Ultrasonic Signals. NASA TM-81489. 
Vary, A. and Hull, D.R. (1982), Interrelation of Material Microstructure, Ultrasonic Factors, and Fracture Toughness of a Two-Phase Titanium Alloy. Mater. Eval., vol. 41, no. 3, pp. 309-314.

Vary, A. and Hull, D.R. (1983), Ultrasonic Ranking of Toughness of Tungsten Carbide. Proceedings of the 14th Symposium on Nondestructuve Evaluation, D.W. Moore and G.A. Matzkanin, eds., Southwest Research Institute, San Antonio, TX, pp. 212-219.

Vary, A. (1984), Ultrasonic Nondestructive Evaluation, Microstructure, and Mechanical Property Interrelations. NASA TM-86876.

Karagulle, H., Lee, S.S., and Williams, J.H., Jr.(1985a), Input-Output Characterization of an Ultrasonic Testing System by Digital Signal Analysis. Analytical Ultrasonics in Materials Research and Testing, NASA CP-2383.

Williams, J.H., Jr., and Lee, S.S. (1985b), Pattern Recognition Characterization of Micromechnical and Morphological Materials States Via Analytical Quantitative Ultrasonics. Analytical Ultrasonics in Materials Research and Testing, NASA CP-2383.

Winkler, K.W. and Murphy, W.F., III (1984), Scattering in Glass Beads: Effects of Frame and Pore Fluid Compressibilities. J. Acoust. Soc. Am., vol. 76 , no. 3, pp. 820-825.

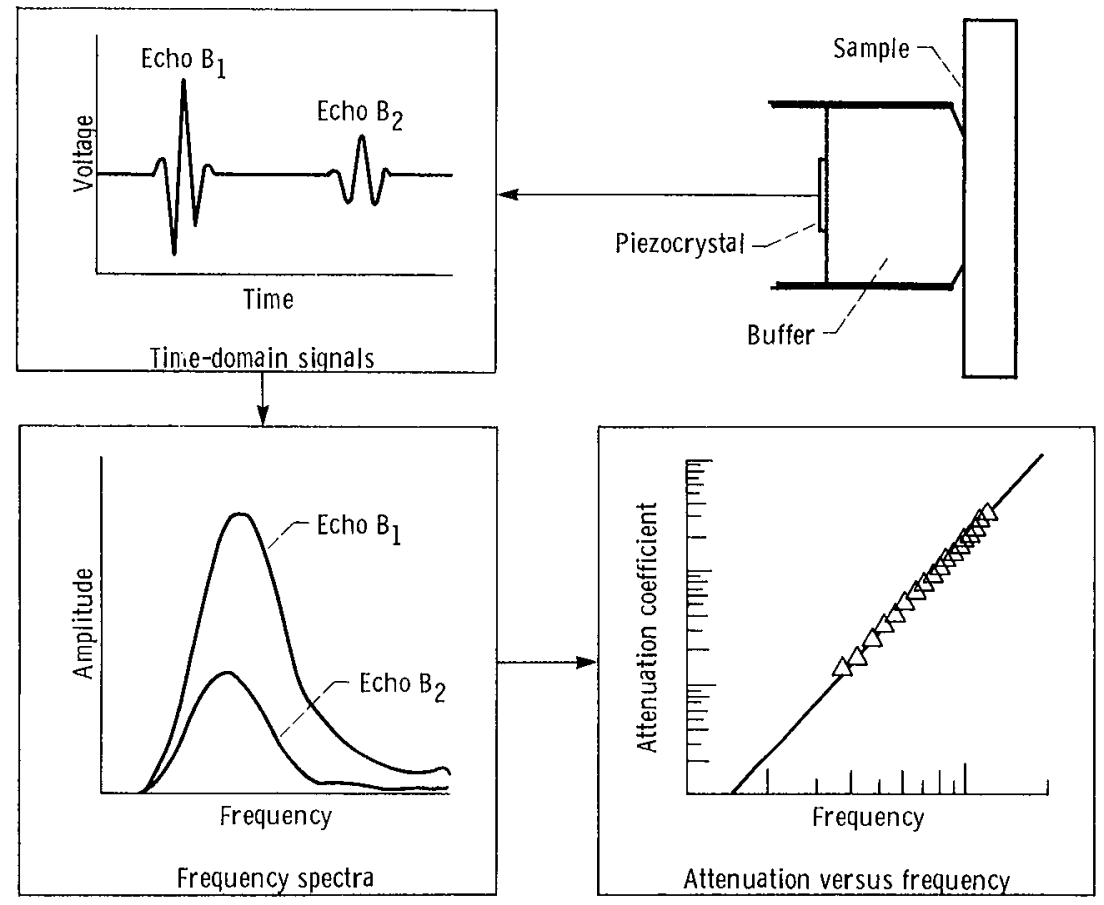

Figure 1. - Schematic of signal acquisition and data-processing stages for determining frequency dependence of attenuation coefficient by using broadband ultrasonic pulse-echo method. 


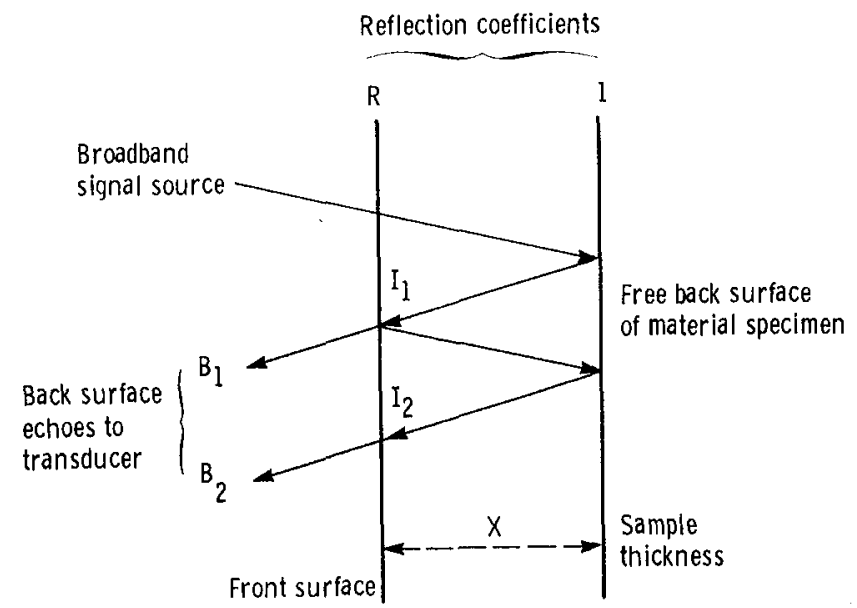

Fiqure 2. - Diagrami of broadband ultrasonic echo system for determining frequency dependence of attenuation coefficient and deriving material microstructure transfer function.

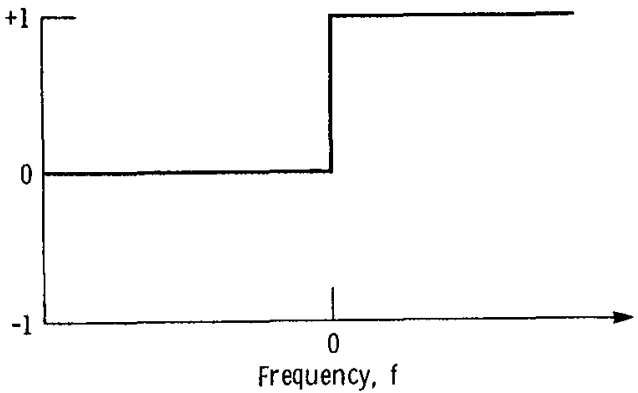

(a) Heaviside step function I $H(f) \mathrm{I}$.

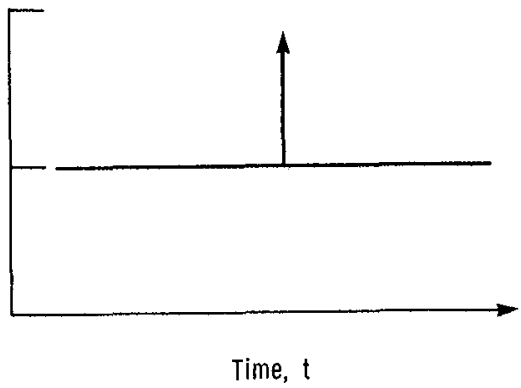

(b) Delta function $\delta(f)$.

Figure 3. - Delta function and Heaviside step function representing time- and frequency-domain counterparts, respectively, of refiection coefficient, where $|R(f)|-|H(f)|-1$ at free surface (appendix B). 


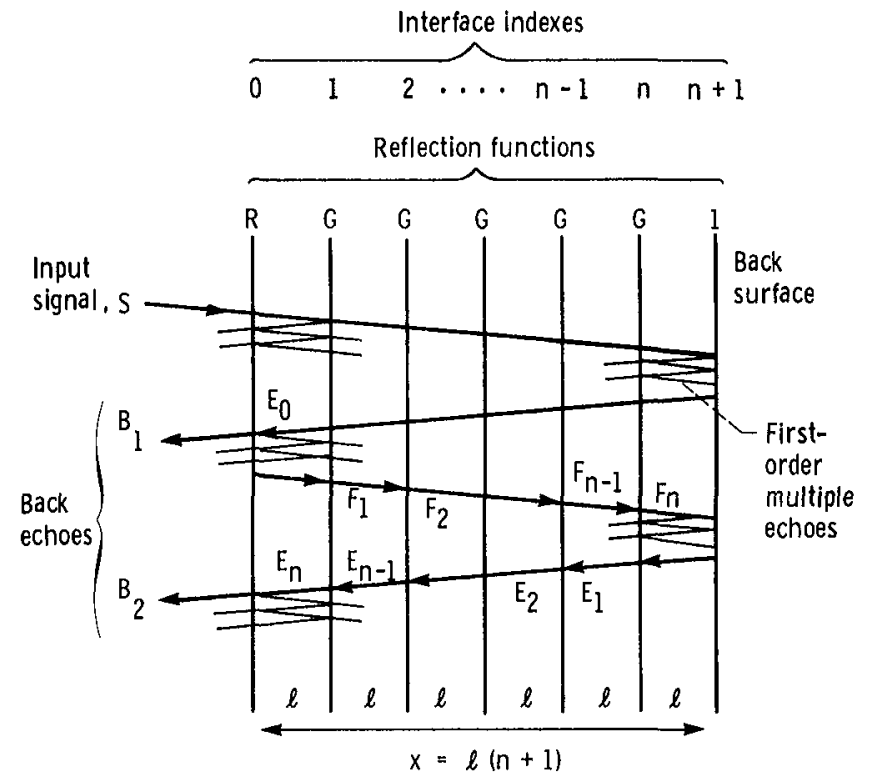

Figure 4. - Diagram of lattice model for synthesizing material structure transfer function (appendix C).

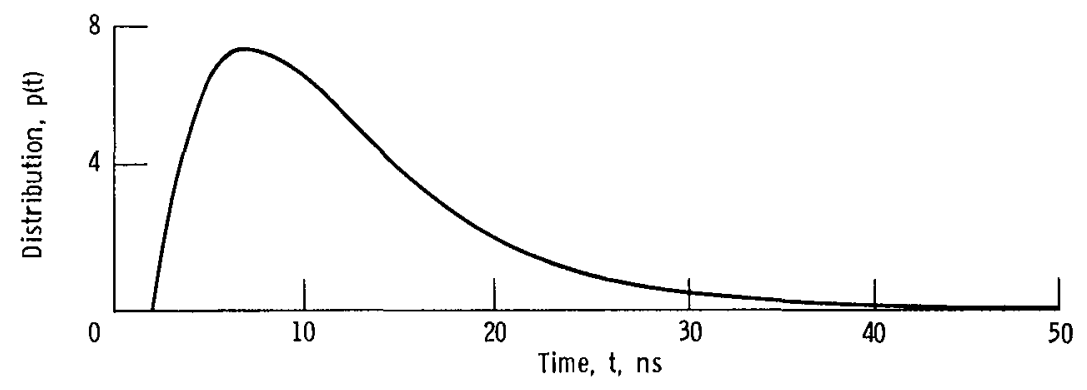

(a) Grain-delay probability distribution function.
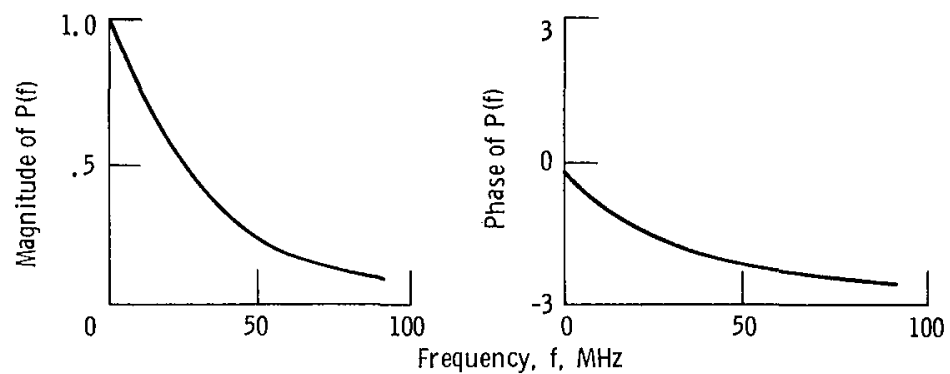

(b) Magnitude and phase spectra of grain-delay distribution function.

Figure 5. - Time-domain probability distribution function for grain-delay (transit time) and its Fourier transform, magnitude, and phase. 


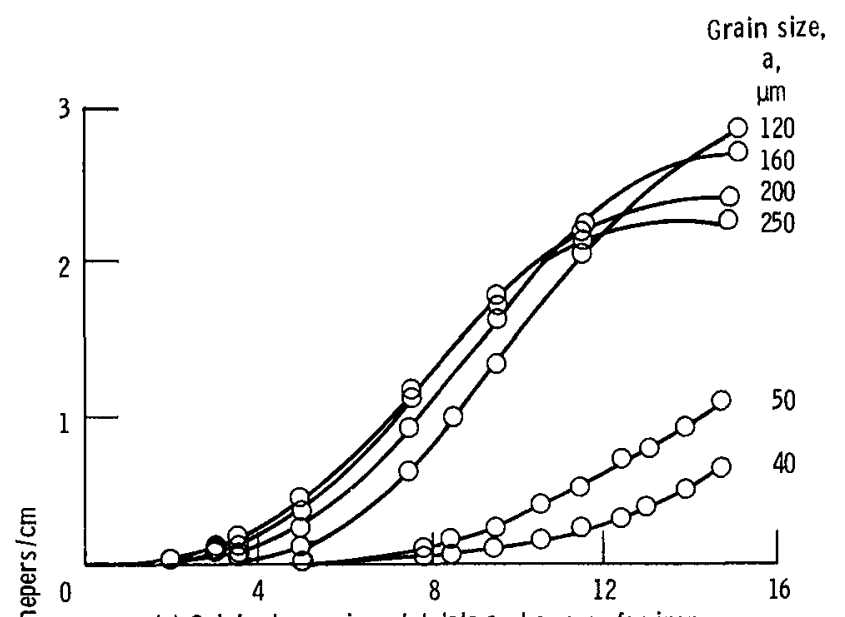

(a) Original experimiental data and curves for iron.

을

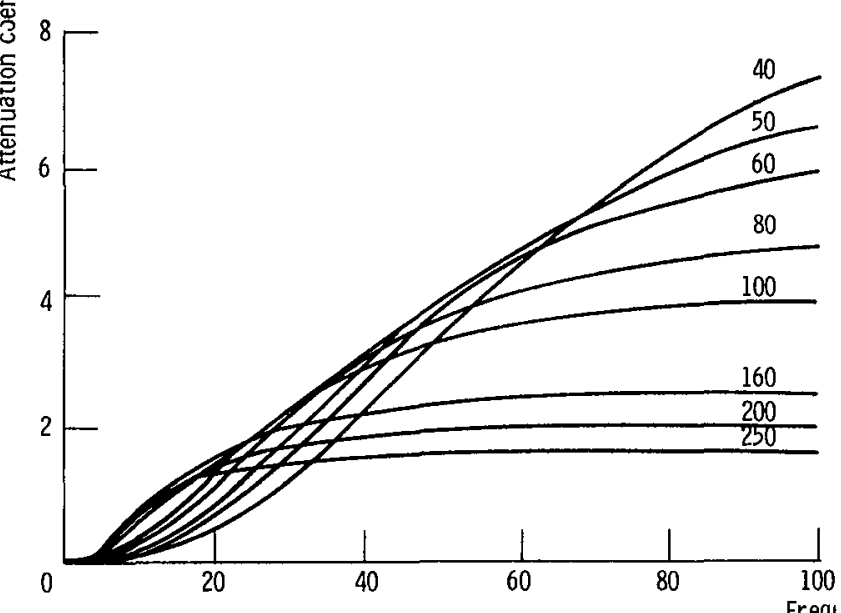

(c) Simulated curves for iron to $100 \mathrm{MHz}(\mathrm{G}=\mathrm{K}=0.0067, \mathrm{~h}=0.00002$ $v=0.59 \mathrm{~cm} / \mu \mathrm{s})$.

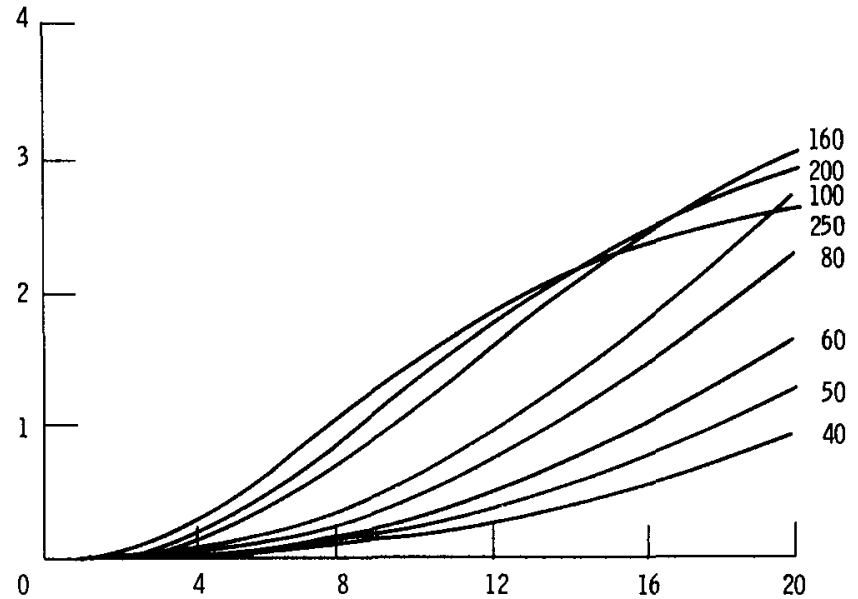

(b) Simulated attenuation data curves for iron $G=K=0.0134$, $h=0.00002, v=0.59 \mathrm{~cm} / \mu \mathrm{s}$.

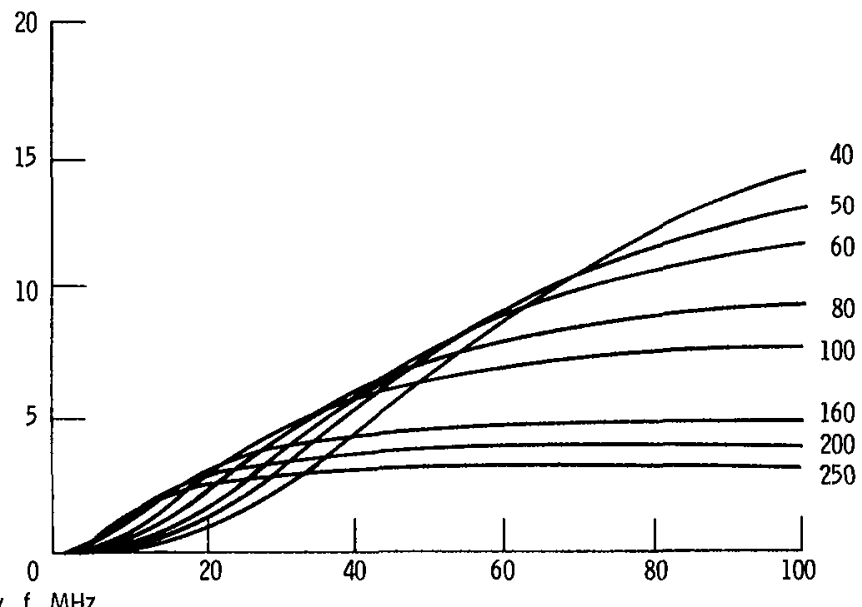

(d) Simulated curves for iron to $100 \mathrm{MHz}$ (G $=K=0.0134, h=0.00002$, $v=0.59 \mathrm{~cm} / \mu \mathrm{s})$.

Figure 6. - Comparison of experimiental attenuation-coefficient-versus-frequency curves for iron with curves generated with equation (34). Oriqinal experimental data fromi Merkulov (1957). 

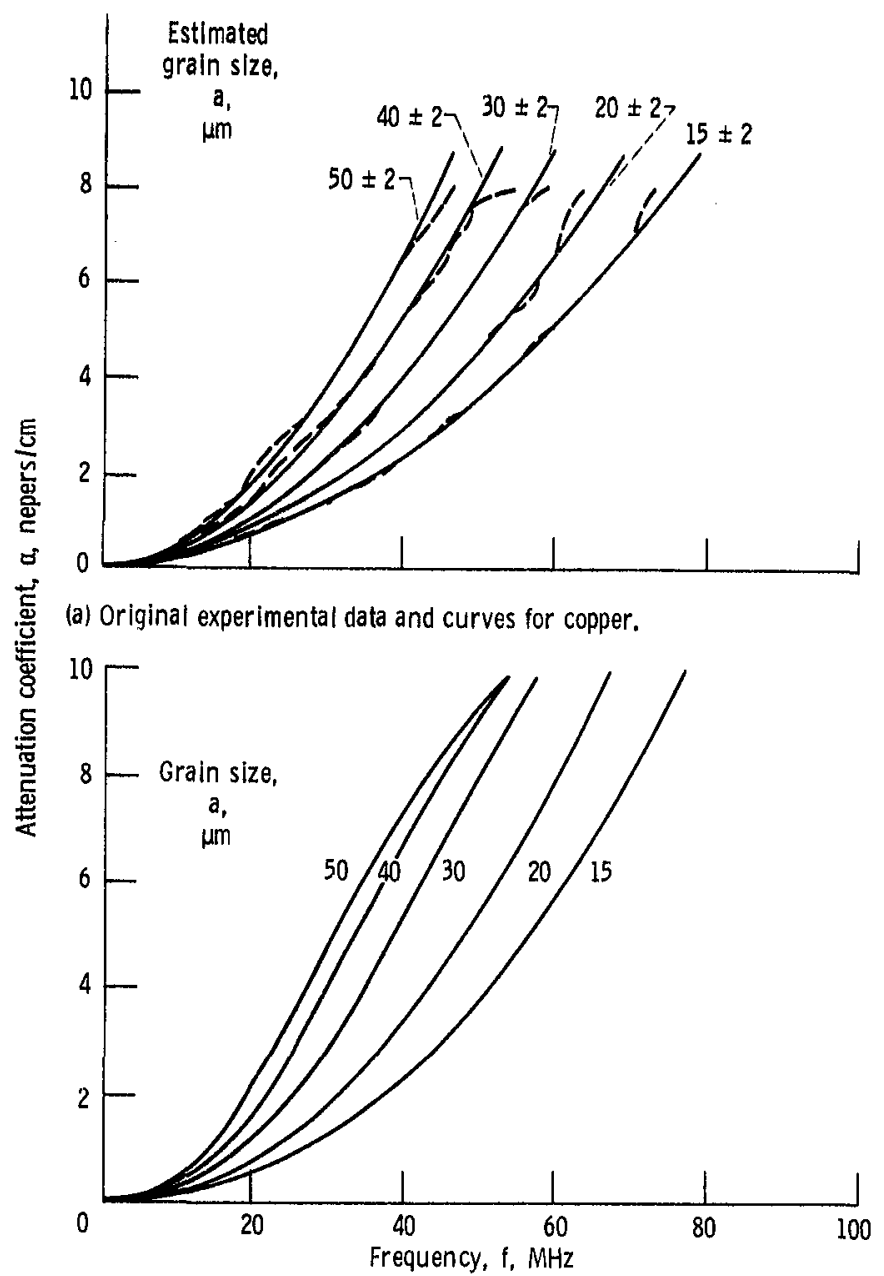

(b) Simulated attenuation data curves for copper $(G=K=0.0124$, $h=0.0003, v=0.47 \mathrm{~cm} / \mu \mathrm{s}$ ).

Figure 7. - Comparison of experimiental attenuation-coefficient-versusfrequency curves for copper with curves generated with equation (34). original experimental data and curves fromı Generazio (1985). 


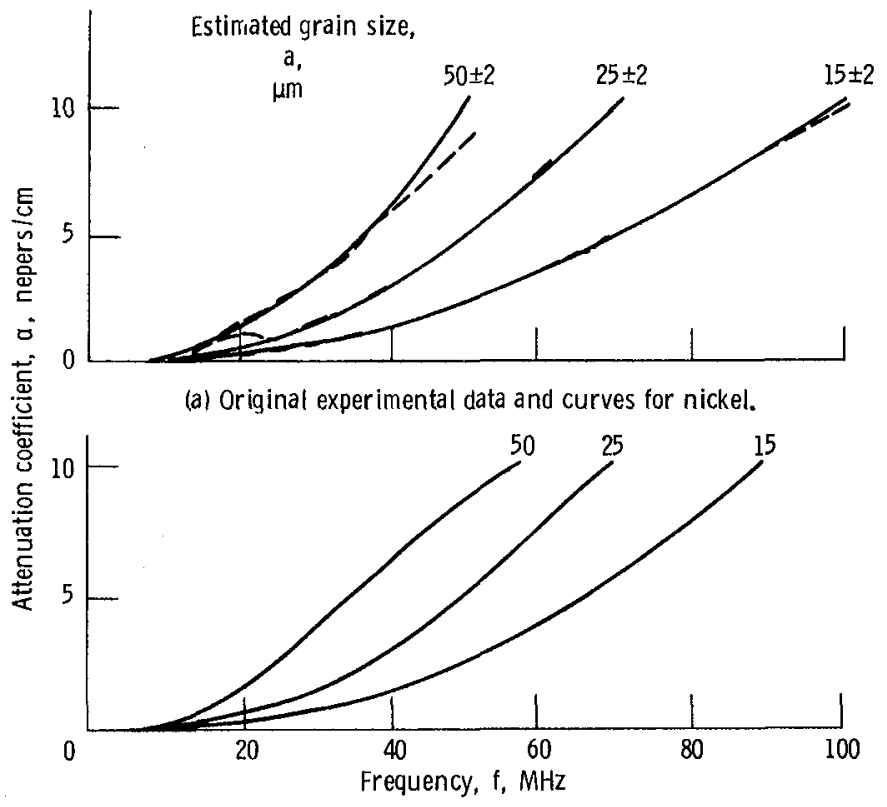

(b) Simulated attenuation data curves for nickel $G=K=0.0144$, $h=0.0003, v=0.563 \mathrm{~cm} / \mu \mathrm{s}$ ).

Figure 8. - Comparison of experimiental attenuation-coefficient-versusfrequency curves for nickel with curves generated with equation (34). Original experimental data and curves from Generazio (1985). 

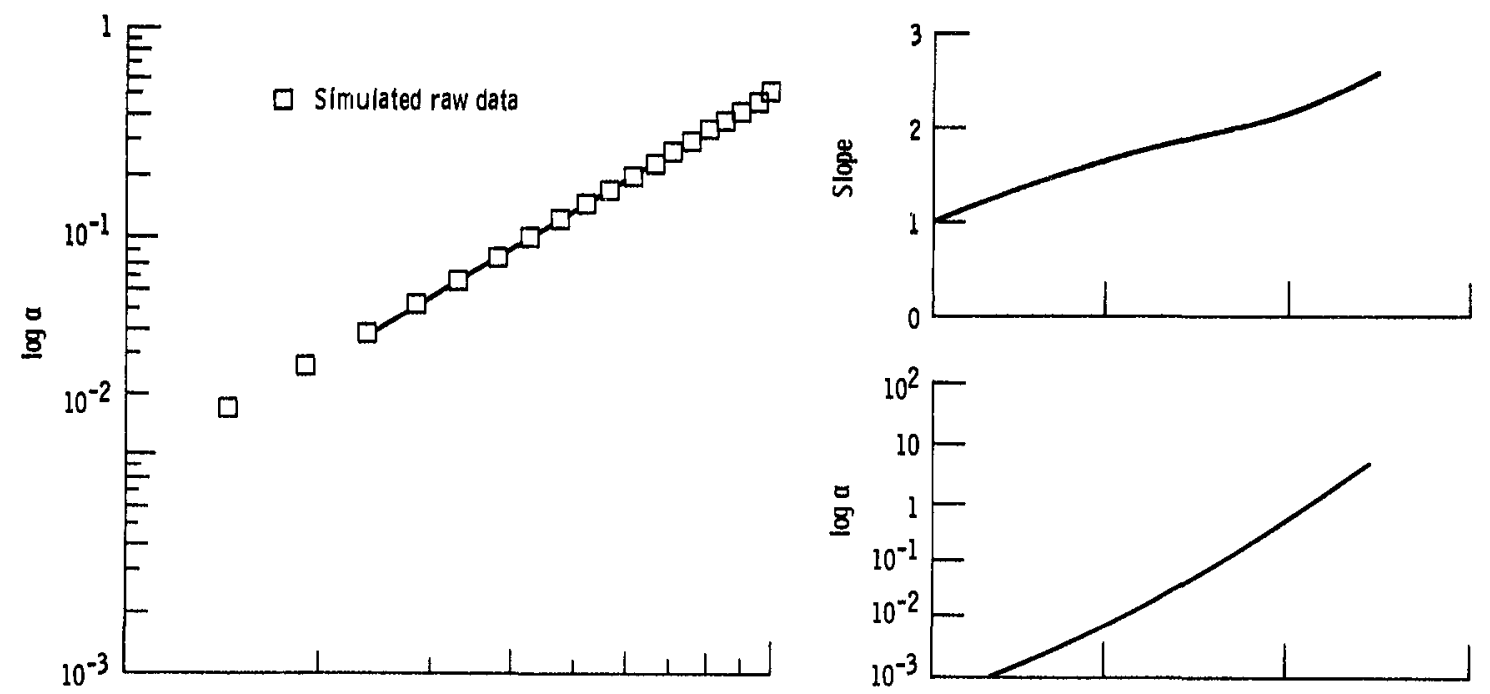

(a) Mean grain size, $2 \mu \mathrm{m} . c=1.016 \times 10^{-4} ; \mathrm{m}-1.835$; least squares coefficient $=0.99954$.
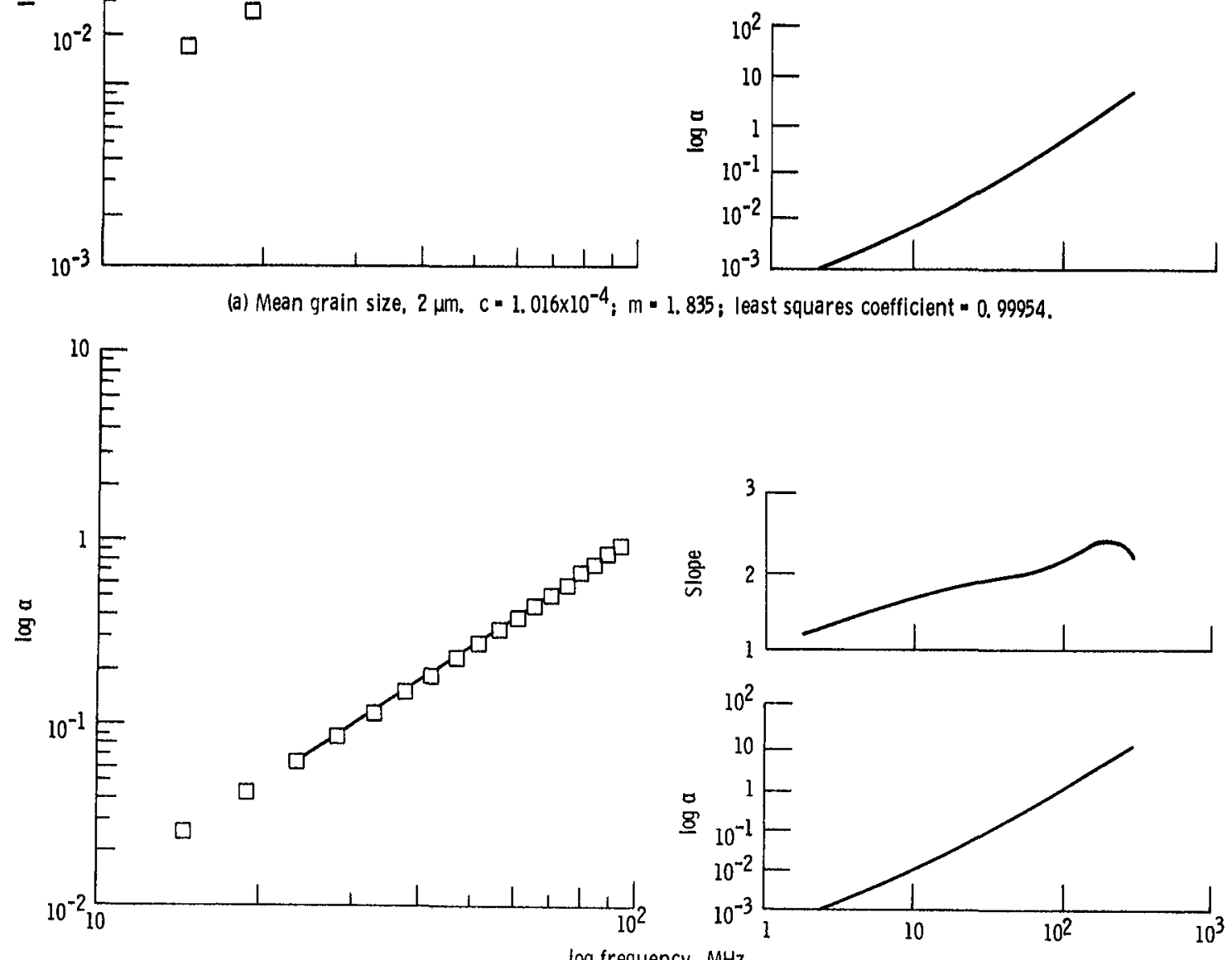

(b) Mean grain size, $4 \mu \mathrm{m}, c=1.143 \times 10^{-4} ; \mathrm{m}=1.975$; least squares coefficient $=0.99947$.

Figure 9. - Simulation of attenuation-coefficient-versus-frequency data in 10- to 100-MHz range using equation (34) and determination of $\mathrm{c}$ and $\mathrm{m}$ values by least squares fit to equation (14) from 15 to $100 \mathrm{MHz}$. $v-0.56 \mathrm{~cm} / \mu \mathrm{s} ; \mathrm{K}=0.007$; $G=0.007 ; h=2.0 \times 10^{-4}$ 

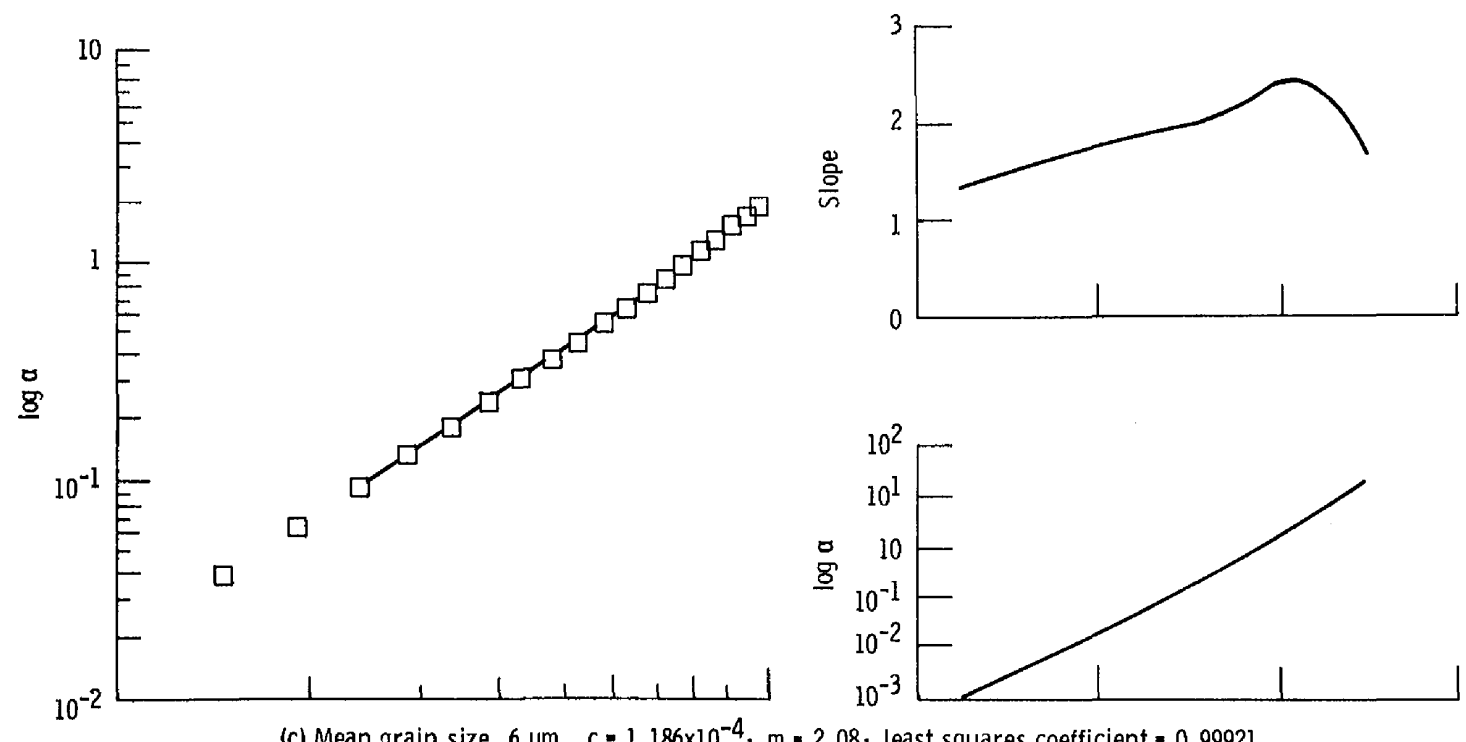

(c) Mean grain size, $6 \mu \mathrm{m}, c=1.186 \times 10^{-4} ; \mathrm{m}=2.08$; least squares coefficient $=0.99921$.
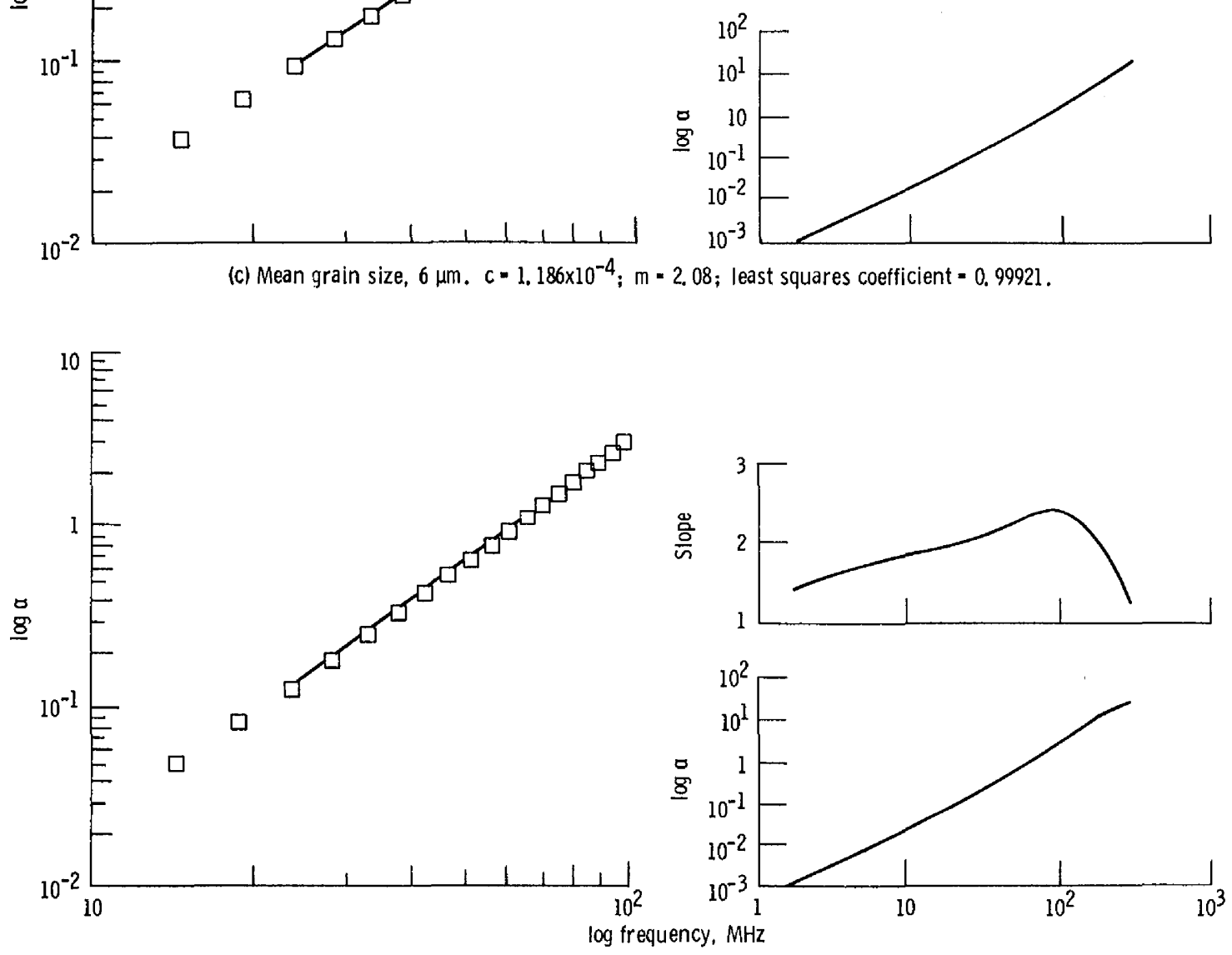

(d) Mean grain size, $8 \mu \mathrm{m}, c=1.200 \times 10^{-4} ; \mathrm{m}=2.166$; least squares coefficient $=0.99906$.

Figure 9. - Continued. 

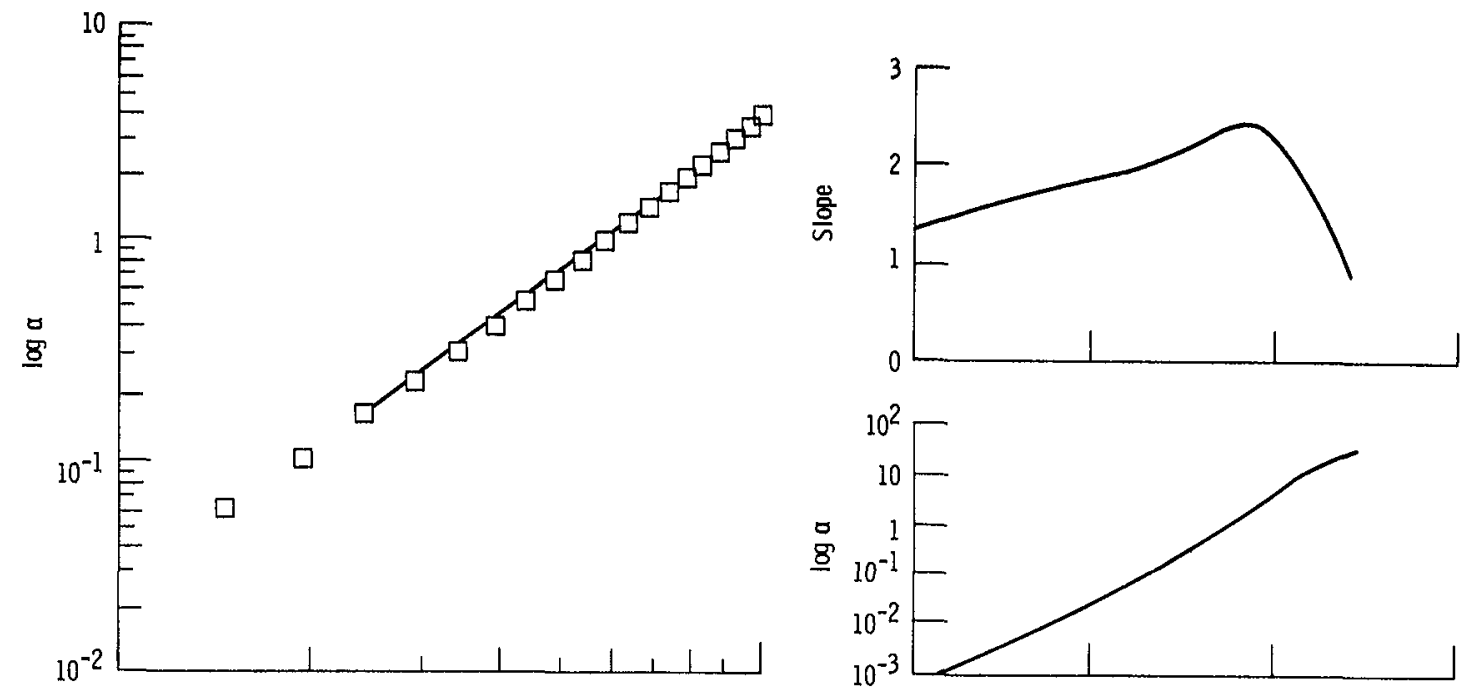

(e) Mean grain size, $10 \mu \mathrm{m}, c=1.238 \times 10^{-4} ; \mathrm{m}=2.231$; least squares coefficient $=0.99913$.
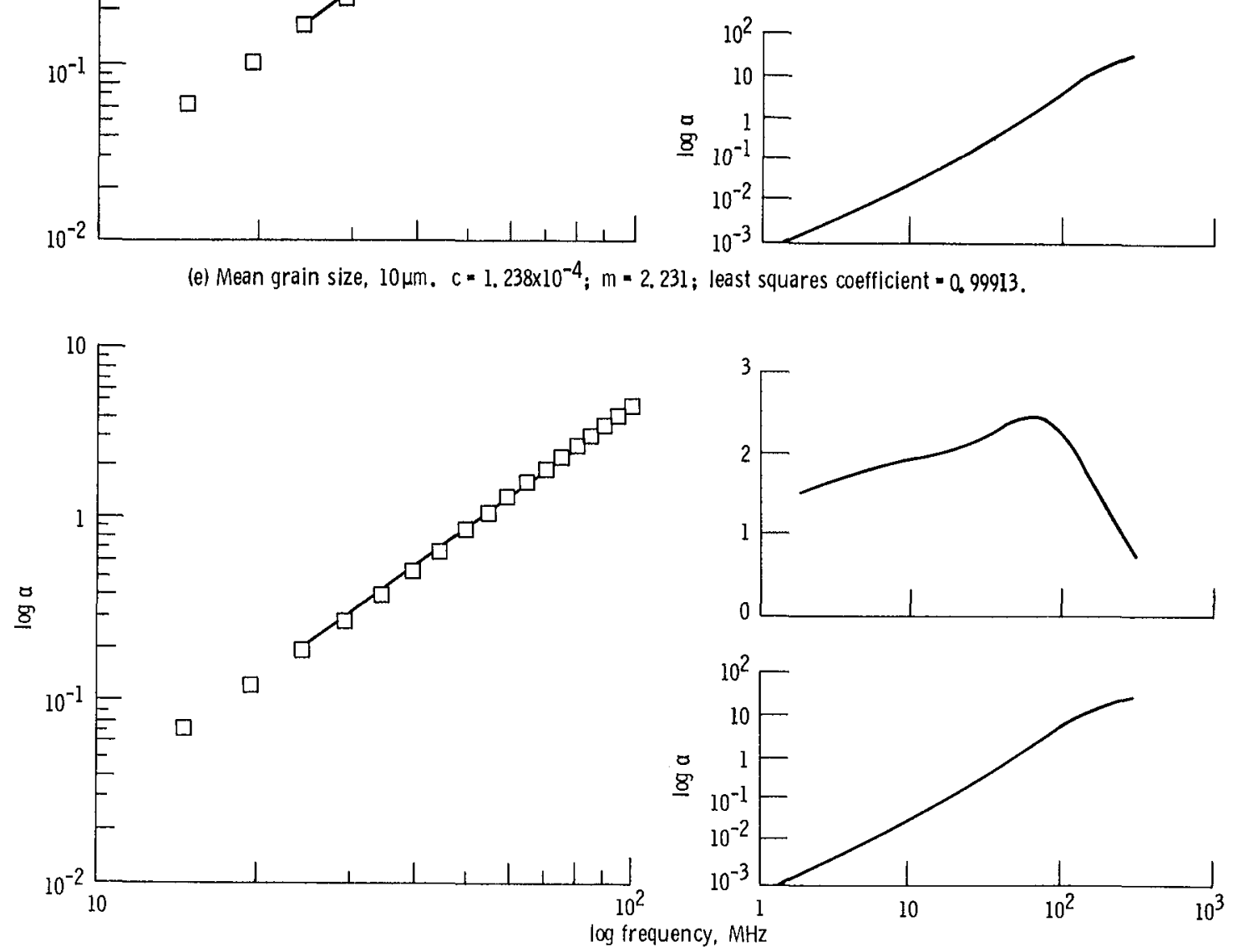

(f) Mean grain size, $12 \mu \mathrm{m} . c=1.328 \times 10^{-4} ; \mathrm{m}=2.274$; least squares coefficient $=0.99935$.

Figure 9. - Continued. 

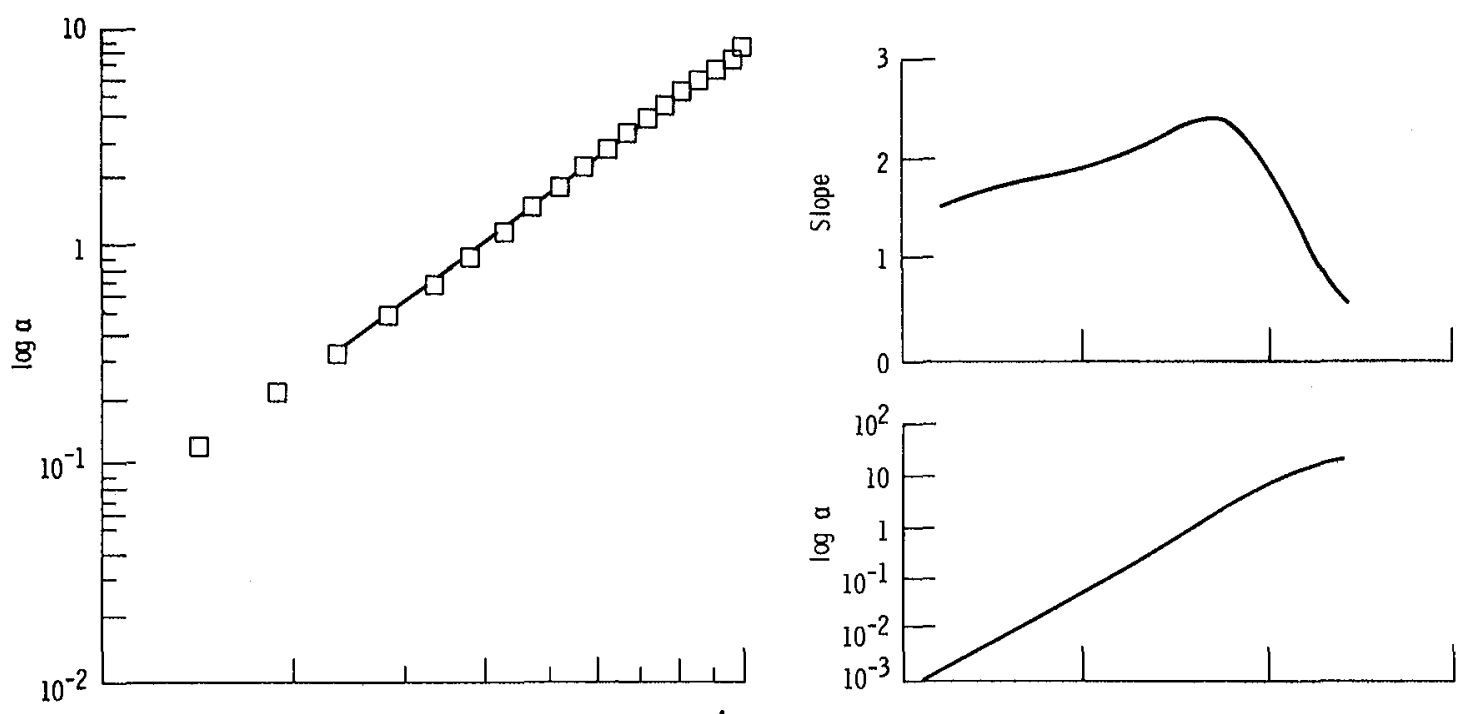

(g) Niean grain size, $14 \mu \mathrm{m} . c=1.487 \times 10^{-4} ; \mathrm{m}=2.297$; least squares coefficient $=0.99958$.
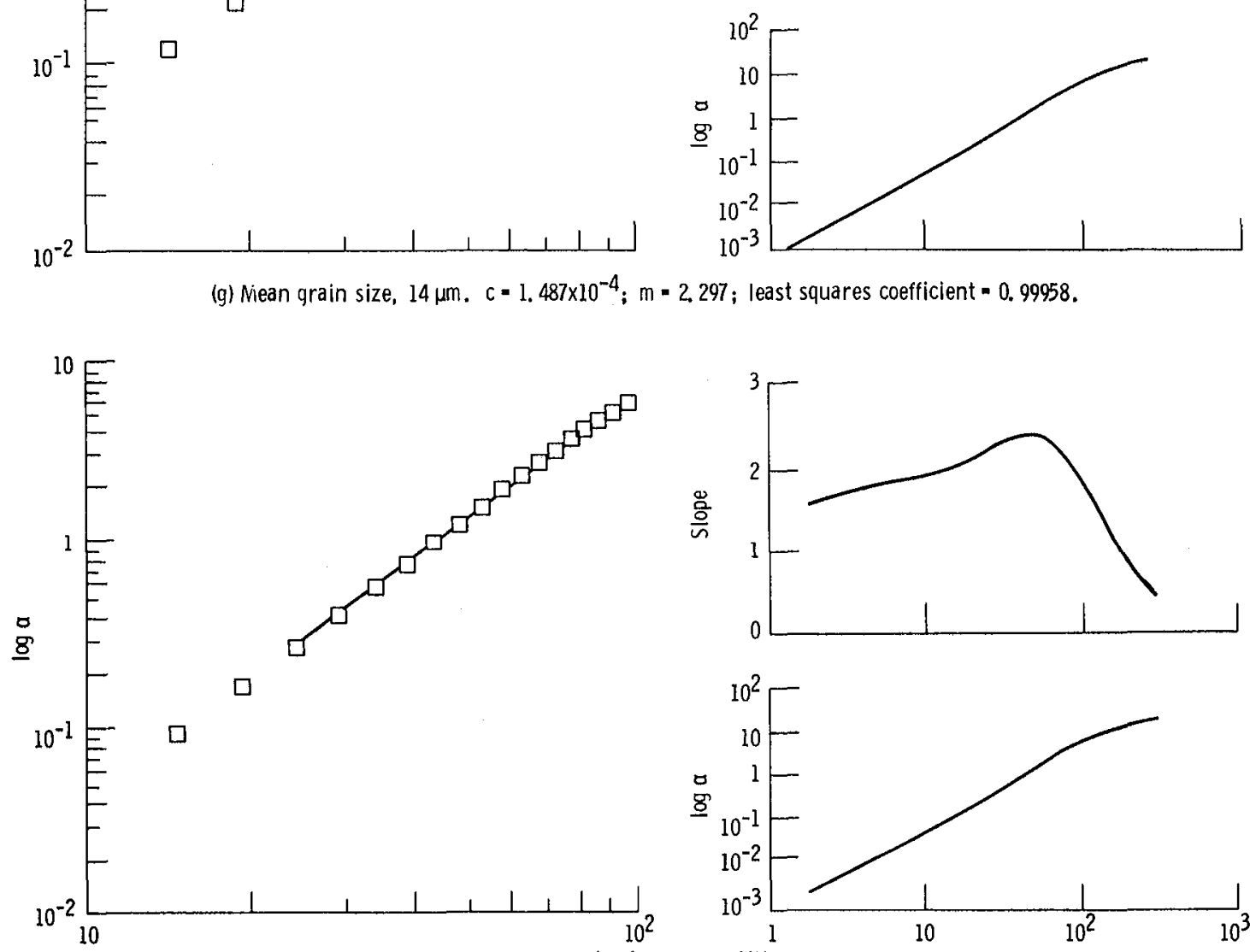

$\log$ frequency, $M H z$

(h) Mean grain size, $16 \mu \mathrm{m} . c=1,729 \times 10^{-4} ; m=2,301$; least squares coefficient $=0.99971$.

Fiqure 9. - Continued. 

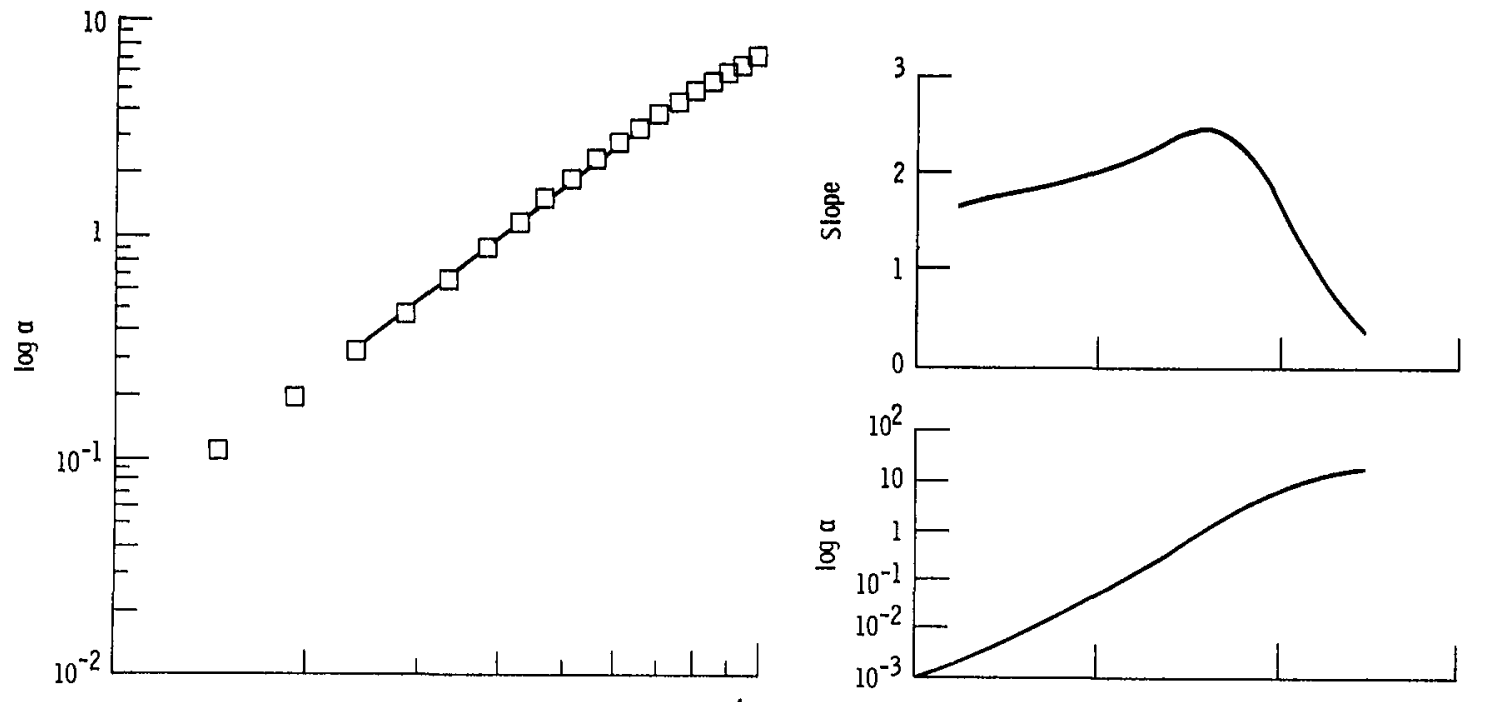

(i) Mean grain size, $18 \mu \mathrm{m} . c=2.077 \times 10^{-4} ; m=2.291$; least squares coefficient $=0.99965$.
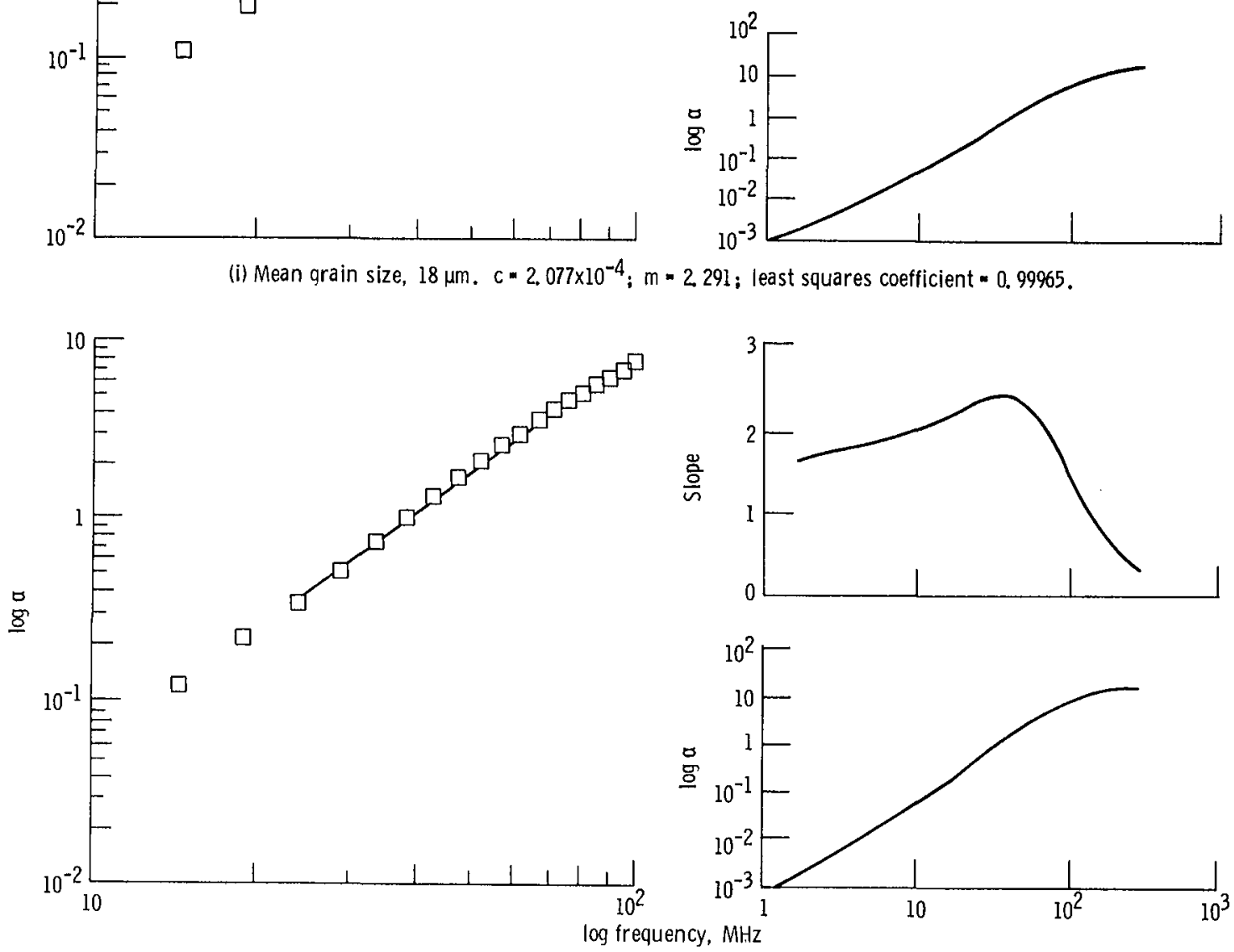

(j) Mean grain size, $20 \mu \mathrm{m} . c=2.556 \times 10^{-4} ; m=2.268$; least squares coefficient $=0.99936$.

Figure 9. - Concluded. 


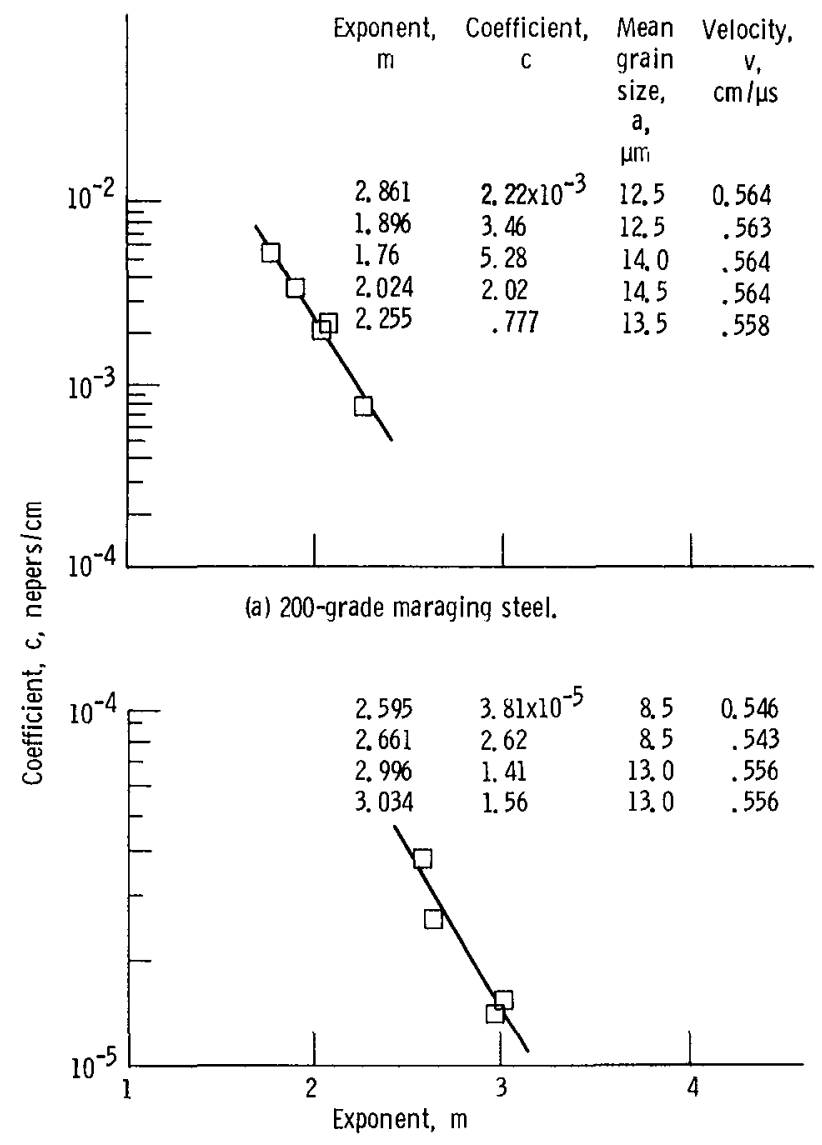

(b) 250-grade maraging steel.

Figure 10. - Inter relations between experimentally determined attenuation parameters $c$ and $m$ from equation (14) for two maraging steels. Data from Vary (1978). 


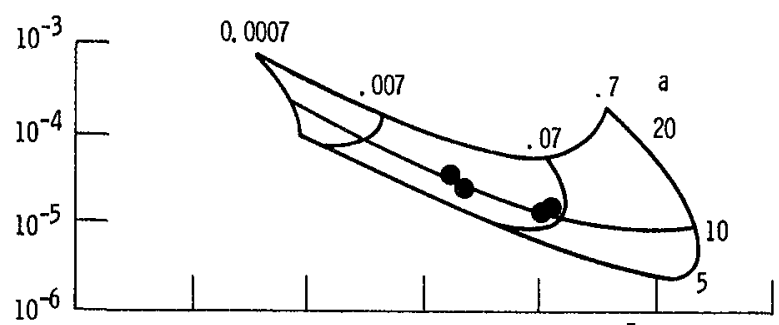

(a) Parametric field for $v=0.55 \mathrm{~cm} / \mu \mathrm{s}, \mathrm{h}=2 \times 10^{-5}, G=0.007$.

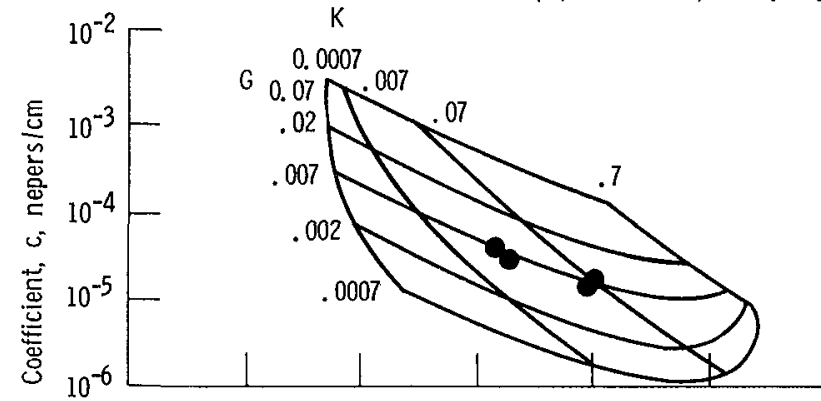

(b) Parametric field for $v=0.55 \mathrm{~cm} / \mu \mathrm{s}, a=10 \mu \mathrm{m}, h=2 \times 10^{-5}$.

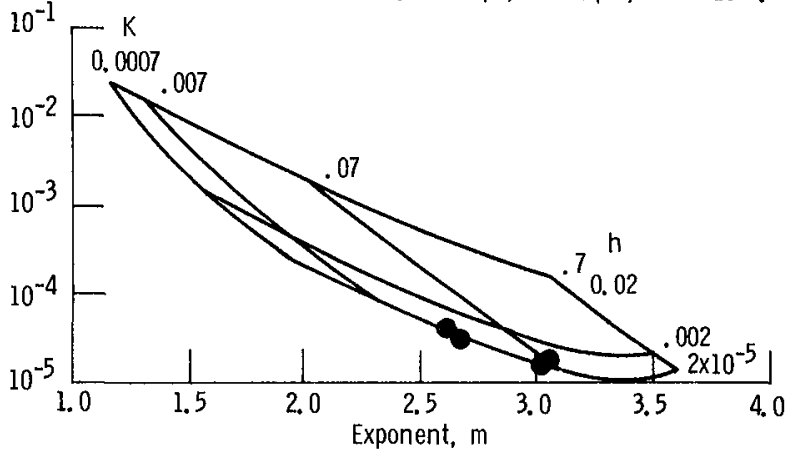

(c) Parametric field for $v=0.55 \mathrm{~cm} / \mu \mathrm{s}, a=10 \mu \mathrm{m}, G=0.007$.

Figure 11. - Parametric fields of $c$ versus $m$ based on equations (14) and (34) for assigned values of mean grain size a, velocity $v$, hysteresis constant $h$, average reflection coefficient $G$, and elastic anisotropy factor $K$. Using the curve-fitting method illustrated in figure 9 the fields contain only points for which the correlation coefficient exceeds 0.999 over the frequency range 15 to $100 \mathrm{MHz}$. Data for 250 -grade maraging steel from figure 10 are coplotted within fields. 